\title{
Quantitative gene profiling of long-noncoding RNAs with targeted RNA sequencing
}

Michael B. Clark ${ }^{1,2,10}$, Tim R. Mercer ${ }^{1,3,10}$, Giovanni Bussotti ${ }^{4}$, Tommaso Leonardi ${ }^{4}$, Katelin R. Haynes ${ }^{5}$, Joanna Crawford ${ }^{6}$, Marion E. Brunck ${ }^{7}$, Kim-Anh Lê Cao ${ }^{5,6}$, Gethin P. Thomas $^{5}$, Wendy Y. Chen ${ }^{1,3}$, Ryan J. Taft ${ }^{6,8,9}$, Lars K. Nielsen ${ }^{7}$, Anton J. Enright ${ }^{4}$, John S. Mattick ${ }^{1,3}$ and Marcel E. Dinger ${ }^{1,3}$

${ }^{1}$ Garvan Institute of Medical Research, Sydney, Australia

${ }^{2}$ MRC Functional Genomics Unit, Department of Physiology, Anatomy, and Genetics, University of Oxford, Oxford, United Kingdom

${ }^{3}$ St Vincents Clinical School, Faculty of Medicine, University of New South Wales, Sydney, Australia

${ }^{4}$ EMBL European Bioinformatics Institute, Hinxton, United Kingdom

${ }^{5}$ The University of Queensland Diamantina Institute, The University of Queensland, Translational Research Institute, Brisbane, QLD, Australia

${ }^{6}$ Institute for Molecular Bioscience, The University of Queensland, Brisbane, Australia

${ }^{7}$ Australian Institute for Bioengineering and Nanotechnology, The University of Queensland, Brisbane, Australia

${ }^{8}$ Illumina, Inc., San Diego, USA

${ }^{9}$ School of Medicine and Health Services, Departments of Integrated Systems Biology and of Pediatrics, George Washington University, USA

${ }^{10}$ Authors contributed equally

Correspondence:

John S. Mattick

Tel: +61 292958120

Marcel E. Dinger

Fax: +61292958101

Tel: +61293555860

Email: j.mattick@garvan.org.au

Fax: +61 292958101

Email: m.dinger@garvan.org.au

We compared the ability of qRT-PCR, RNA sequencing and Capture Sequencing (CaptureSeq) to assemble and quantify long noncoding RNAs and novel coding exons across 20 human tissues. CaptureSeq is superior for detection and quantification of lowly expressed genes, shows little technical variation and accurately measures differential expression. This approach expands and refines previous annotations and simultaneously generates an expression atlas. 
The majority of the human genome is differentially expressed across a wide dynamic range to produce a spectrum of protein-coding and noncoding RNAs, generating a transcriptome of unexpected scale and complexity ${ }^{1-3}$. These features present a challenge for gene expression profiling with quantitative RT-PCR (qRT-PCR) and RNA sequencing (RNAseq). qRT-PCR is ill-suited for gene discovery, resolving alternative splicing or characterizing whole transcriptomes (Supplementary Fig. 1a). RNAseq measures global gene abundance and splicing, but provides scant coverage of rare transcripts, limiting the sensitivity of transcript assembly and quantification (Supplementary Fig. 1b,c) ${ }^{1,4}$. We recently developed CaptureSeq, which enriches transcripts of interest by hybridization to magnetic bead-linked oligonucleotides that are tiled across the region of interest, allowing targeted purification, multiplexed library preparation and RNA sequencing at high depth ${ }^{5,6}$. (Supplementary Fig. 1d). .

To assess CaptureSeq's quantitative accuracy, we undertook an analysis of transcript quantification in direct comparison to conventional RNAseq and qRT-PCR. As an independent reference, External RNA Control Consortium (ERCC) RNA standards spanning a $\sim 10^{6}$-fold range of concentrations were spiked into three biological replicate human K562 RNA samples ${ }^{4,7}$. We then performed CaptureSeq on each sample, targeting the full range of standards, along with matched RNAseq. CaptureSeq exhibited high correlation between biological and technical replicates (mean Spearman's $r \geq 0.998$ in measured ERCC abundance; Supplementary Fig. 2b,c) similar to matched RNAseq (Spearman $r=0.991$ ) (Supplementary Fig. 2d).

The measurement of ERCC standards using CaptureSeq exhibits a segmented profile, with an inflection point at $\sim 2.34$ attomoles/ $\mu$ l (Fig. 1a, and Supplementary Fig. 2e, Supplementary Results). Above this point, CaptureSeq increasingly underestimates the abundance of ERCC standards, likely due to the saturation of oligonucleotide capture-probes. By contrast, RNAseq retains linear quantification and provides accurate measurement of high abundance ERCC standards (Fig. $\mathbf{1 b}$ and Supplementary Fig. 2f). Below this point, CaptureSeq achieves a mean 157 fold enrichment in coverage and as a result measures low abundance standards (Pearson $\mathrm{R}^{2}=0.896$ from 0.00014-0.0183 attomoles/ $\mu \mathrm{l}$ ) more accurately than RNAseq (Pearson $R^{2}=0.753$; (Fig. 1 and Supplementary Fig. 2a).

An eight-fold sequence coverage is the estimated lower limit at which transcript models can be confidently assembled ${ }^{4}$. CaptureSeq achieves this for all ERCC concentrations tested, corresponding to the assembly of as little as $\sim 1,550$ transcripts in the input RNA (Supplementary Fig. 3a,b). By contrast RNAseq was unable to reliably detect low ERCC concentrations $(<0.0366$ attomoles $/ \mu \mathrm{l})$, precluding the accurate measurement of low abundance standards (Supplementary Fig. 3c,d).

RNAseq and CaptureSeq quantified moderately abundant standards (0.0366-2.34 attomoles/ $\mu$ l) comparably well (Fig. 1a,b). Therefore, by comparing this subset of matched ERCC standards, we can discern biases resulting from the additional steps of hybridization, elution, washing and PCR amplification used in CaptureSeq but not RNAseq (Supplementary Results, Supplementary Fig. 4). We find these steps collectively contribute only minor technical variation ( $4.8 \%$ of total), with systematic 
biases inherent to library preparation and sequencing being the dominant source of error $^{8,9}$.

We next measured the fidelity with which CaptureSeq measures expression differences. We captured and sequenced two distinct pools of ERCC standards mixed at different relative concentrations (Supplementary Results, Supplementary Fig. 5ae). CaptureSeq detected but slightly underestimated fold changes between the pools (Fig. 1e). Notably, CaptureSeq achieved an accurate appraisal of differential gene expression of two-fold or greater across the full range of ERCC standards and outperformed RNAseq at quantifying differential expression of low abundance transcripts (Supplementary Fig. 5f and 6).

We also compared qRT-PCR to CaptureSeq. We performed qRT-PCR to measure the abundance of nine ERCC probes over a $2.5 \times 10^{5}$ - fold range. As with RNAseq, we found qRT-PCR measures the lowest abundance standards less accurately than CaptureSeq (Fig. 1c), but is equal or superior at higher abundances.

To provide a context to the advantages of CaptureSeq, we compared the concentrations of ERCC standards to the endogenous RNA population from K562 cells. We estimate that $42.1 \%$ of RNA transcripts are better quantified using CaptureSeq (Fig. 1d), RNAseq and CaptureSeq perform comparably well for $53.2 \%$ of transcripts, and RNAseq performs better for the top $4.6 \%$ expressed transcripts that are enriched for housekeeping, structural and metabolic genes. By contrast, lowly expressed genes in K562 cells for which CaptureSeq provides superior quantitative accuracy are enriched for transcription factors, genes associated with cancer and human disease genes (Supplementary Fig. 7a-c).

To demonstrate the advantages of CaptureSeq for gene discovery and quantitative profiling, we focused on IncRNAs, which are often poorly annotated, lowly expressed and highly tissue-specific ${ }^{10,11}$. CaptureSeq probes were designed to target all publicly annotated IncRNAs comprising 29,127 different transcripts that constitute $\sim 7.9 \%$ of the K562 transcriptome (Fig. 2a, Supplementary Data 1$)^{10,12,13}$. The efficacy of capture design was initially tested by DNA hybridization and sequencing, and a suite of internal controls were included to measure hybridization performance (Supplementary Results, Supplementary Fig. 7d,e).

We initially performed IncRNA CaptureSeq using two K562 replicate samples with accompanying matched RNAseq. We achieved 10.45 -fold enrichment as determined from enrichment of ERCC controls (83\% of the possible 12.6-fold enrichment; Fig. 2b). By selectively targeting exons we enriched for spliced transcripts, observing a 2.2fold reduction in nascent transcription observed within introns and a 1.83-fold enrichment in informative spliced reads that traverse exon-exon junctions relative to conventional RNAseq, thereby aiding better assembly with less contamination from unspliced reads (Supplementary Fig. 7f).

We subsequently expanded our application of CaptureSeq to profile the expression of IncRNAs across 20 human tissues and 4 cell lines. The quantitative accuracy of each library was confirmed by analysis of ERCC controls. However, despite the enhanced sensitivity conferred by targeted sequencing, we do not achieve routine 
coverage of the lowest abundance ERCC standards (Supplementary Fig. 8), indicating sequencing depth has not yet reached saturation and further transcriptional complexity may remain to be identified.

Almost all targeted exons (89.97\%) were expressed in at least one of the tissues sampled. CaptureSeq also detected an additional 22,046 novel exons (15.9\% more than initial annotations (from GENCODE v19 ${ }^{12}$, Cabili et $\mathrm{al}^{10}$., and $\operatorname{LncRNAdb}^{13}$ ) and 10,163 novel splice junctions ( $9.7 \%$ more), which exhibit similar conservation and splice motif enrichment to previous annotations (see Supplementary Results; Supplementary Fig. 9a,b). CaptureSeq found alternative splicing involves $40.3 \%$ of exons (compared to $26.3 \%$ previously) and thereby illuminates greater isoform diversity (3.6 isoforms per locus compared to 1.8 previously ${ }^{12}$ ). We assembled exons into 54,972 full-length transcript models from 15,272 loci, a 1.8-fold expansion on previous annotations.

The protein-coding potential of transcript models was assessed by ORF length and codon usage (Supplementary Fig. 9d,e) ${ }^{14}$. We identified 13,796 loci that generate 45,399 IncRNA isoforms (17,803 known, 27,596 novel), with $20.6 \%$ more exons and $13.5 \%$ more introns relative to previous annotations. Novel IncRNA exons identified with CaptureSeq exhibited a similar conservation profile, SNP, repeat density and RNA structure profiles as for previous IncRNA annotations (Supplementary Fig. 10). The enhanced coverage enabled the assembly of more complex structures, with a median 4 exons for assembled IncRNAs (compared to 2 exons in previous annotations; Figure S9f). Indeed, 684 previous IncRNAs annotations were found to merge into 234 larger transcriptional units when profiled with CaptureSeq, revealing some notably large and complex IncRNA loci (Fig. 2c, Supplementary Fig. 9c and Supplementary Fig. 11a,b). By clustering of IncRNA expression levels across human tissues we also identify co-expressed subsets of IncRNAs (Supplementary Fig. 12), providing a greatly expanded atlas of IncRNA expression.

The enhanced coverage provided by CaptureSeq enables more complete transcript models that can correct previous erroneous annotations. We found 267 loci (comprising 703 isoforms) previously classified as IncRNAs contained substantial open reading frames. These loci had no overlap with known coding gene transcripts and may represent new putative protein-coding loci. Indeed, 135 transcripts share significant homology with known protein domains ${ }^{15}$, including a 1021 amino acid protein with a highly conserved coiled-coil domain (Supplementary Fig. 11c).

The more complete transcript models provided by CaptureSeq also revealed that some previously annotated IncRNAs comprise distal exons of protein-coding genes (Supplementary Fig. 11d). Expanded annotation with CaptureSeq found 2,177 captured isoforms from 782 loci shared a splice junction with neighboring proteincoding genes (Supplementary Results). The majority (78.5\%) of these spliced transcripts retain their open reading frame and add 1,255 new exons into proteincoding gene models. New exons exhibit slightly lower conservation relative to previous annotations, but exhibit characteristic three-nt codon periodicity (Supplementary Fig. 10a,b) ${ }^{16}$. A notable example found that two IncRNA loci are spliced together with three neighboring protein-coding genes into a single large 64exon transcript containing a 3,188aa open reading frame (Fig. 2d). 
Previously, a comparative analysis of mammalian genomes predicted the existence of 2,060 novel protein-coding exons in non-transcribed regions, based on the evolutionary signature of open reading frames ${ }^{17}$. Using CaptureSeq, we confirmed the expression of $543(26 \%)$ of these exons, including novel exons for 208 proteincoding genes, and identified 16 lincRNAs, plus 10 novel loci, with protein-coding capacity (Supplementary Results, Supplementary Fig. 13).

We have employed CaptureSeq to generate a IncRNA catalogue as a resource for further research (Supplementary Data 2-9). Given its demonstrated advantages over conventional RNAseq, we anticipate that targeted RNA sequencing will become a standard technique for gene expression profiling in both research and the clinic. 
Accession Codes Sequenced libraries have been deposited to GEO as follows. Conventional RNAseq and CaptureSeq in K562 cell line (GSE52503). CaptureSeq of IncRNA annotations in human tissues and cell lines (GSE61474).

Acknowledgments The authors would like to thank the following funding sources: Australian National Health and Medical Research Council (NHMRC) Australia Fellowship (631668 to J.S.M. and 631542 to M.E.D.); NHMRC Early Career Fellowship (APP1072662) and EMBO Long Term Fellowship (ALTF 864-2013) to M.B.C.; Queensland State Government (National and International Research Alliance Program; to L.K.N). EMBL Interdisciplinary Postdoc (EIPOD) under Marie Curie Actions (COFUND) (to G.B.). The contents of the published material are solely the responsibility of the administering institution, a participating institution or individual authors and do not reflect the views of NHMRC. The authors would also like to acknowledge and thank the provision of data by the ENCODE consortium. Data was employed in strict accordance with associated data release policy. Authors would also like to thank Prof. Matt Brown (University of Queensland) for contributions to manuscript preparation.

Author Contributions T.R.M. and M.B.C. conceived project and designed experiments, with advice from M.E.D and J.S.M. M.B.C, J.C, M.E.B and K.R.H. performed RNAseq and RNA CaptureSeq. T.R.M., M.B.C, T.L. and G.B. performed the bioinformatic analyses. K.L.C. assisted with statistical analyses. T.R.M., M.B.C., M.E.D, L.K.N. and J.S.M. prepared the manuscript. M.A.B, G.P.T, A.J.E., L.K.N., R.J.T. M.E.D and J.S.M provided funding.

Conflict Of Interest. T.R.M. is recipient of a Roche Discovery Agreement (2014). M.B.C. has received research support from Roche/Nimblegen for an unrelated research project. 


\section{REFERENCES}

1. Clark, M.B. et al. PLoS Biol 9, e1000625 (2011).

2. Kapranov, P., Willingham, A.T. \& Gingeras, T.R. Nat Rev Genet 8, 413-423 (2007).

3. Djebali, S. et al. Nature 489, 101-108 (2012).

4. Jiang, L. et al. Genome Res 21, 1543-1551 (2011).

5. Mercer, T.R. et al. Nat Protoc 9, 989-1009 (2014).

6. Mercer, T.R. et al. Nat Biotechnol 30, 99-104 (2012).

7. ERCC Consortium. BMC Genomics 6, 150 (2005).

8. Hansen, K.D., Brenner, S.E. \& Dudoit, S. Nucleic Acids Res 38, e131 (2010).

9. Roberts, A., Trapnell, C., Donaghey, J., Rinn, J.L. \& Pachter, L. Genome Biol 12, R22 (2011).

10. Cabili, M.N. et al. Genes Dev 25, 1915-1927 (2011).

11. Derrien, T. et al. Genome Res 22, 1775-1789 (2012).

12. Harrow, J. et al. Genome Res 22, 1760-1774 (2012).

13. Amaral, P.P., Clark, M.B., Gascoigne, D.K., Dinger, M.E. \& Mattick, J.S. Nucleic Acids Res 39, D146-151 (2011).

14. Wang, L. et al. Nucleic Acids Res 41, e74 (2013).

15. Finn, R.D. et al. Nucleic Acids Res 42, D222-230 (2014).

16. Keren, H., Lev-Maor, G. \& Ast, G. Nat Rev Genet 11, 345-355 (2010).

17. Lindblad-Toh, K. et al. Nature 478, 476-482 (2011).

18. FANTOM Consortium. Nature 507, 462-470 (2014).

19. ENCODE Consortium. Nature 489, 57-74 (2012).

20. Bernstein, B.E. et al. Nat Biotechnol 28, 1045-1048 (2010). 


\section{FIGURE LEGENDS}

Figure 1. Quantitative comparison of CaptureSeq, RNAseq and qRT-PCR. (a-c) Scatterplots indicate correlations between ERCC-standards abundance and measured RPKM for (a) CaptureSeq, (b), RNAseq, and (c) qRT-PCR (from cDNA); n=3 $(a, b), n=2$ (c) biological replicates, error bars indicate standard deviation (s.d). Boxes indicate abundance (low: red, medium: purple, high: blue). (d) Frequency distribution contextualizes the relative performances of gene profiling techniques in relation to endogenous gene expression in K562 human cells. Approximately $42.1 \%$ of transcripts are expressed at levels corresponding to low abundance ERCC standards for which CaptureSeq exhibits better quantitative performance; $n=3$ biological replicates, error bars indicate s.d. (e) Scatterplot shows fold-change quantification of ERCC standards between two distinct samples (ERCC Mix no.1; $n=5$ and Mix no.2; $\mathrm{n}=4$ biological replicates).

Figure 2. Profiling of human long noncoding RNAs with CaptureSeq. (a) Schematic diagram of CaptureSeq for transcriptional profiling. (b) Frequency distribution of total (black), coding mRNAs (blue) and IncRNAs (red) in K562 cells. (c) Three original IncRNA annotations (blue) are merged into larger spliced transcripts following targeted sequencing and enhanced coverage provided by CaptureSeq. Arrows indicate direction of transcription (d) Enhanced coverage provided by targeted sequencing enables two IncRNA and three coding genes to merge into a single locus with a large predicted ORF. Also indicated are transcriptional start sites and enhancer positions from FANTOM $5^{18,19}$, H3K4me3 sites from Roadmap Epigenome H3K4me3 summary for 121 sample types ${ }^{20}$, and RT-PCR primers used to confirm assembly. 


\section{ONLINE METHODS}

\section{Cell line culture and RNA extraction}

Human K562, MCF-7 and SK-N-SH cells were purchased from Cellbank Australia. All cell lines were cultured at $37^{\circ} \mathrm{C}, 5 \% \mathrm{CO}_{2}$ with ten percent fetal bovine serum and penicillin/streptomycin. K562 and HeLa cells were cultured in RPMI and DMEM respectively, MCF-7 and SK-N-SH cells were cultured in MEM. Briefly, RNA was extracted by TRIzol, DNase treated with Turbo ${ }^{\mathrm{TM}}$ DNase (Life Technologies), confirmed DNA-free ${ }^{5}$ and its integrity confirm by Agilent 2100 Bioanalyzer (Agilent Technologies). $5 \mu \mathrm{g}$ quantities of RNA were ribodepleted with Ribo-Zero ${ }^{\text {TM }}$ (Epicentre). Samples with low RIN values or which showed little RNA remaining after rRNA depletion were excluded and sample preparation repeated.

\section{Human tissue panel}

FirstChoice Tissue Panel (Life technologies) was utilized to examine 20 human tissues. $5 \mu \mathrm{g}$ quantities of RNA were ribodepleted with Ribo-Zero ${ }^{\mathrm{TM}}$ (Epicentre) and successful rRNA removal validated with Agilent 2100 Bioanalyzer (Agilent Technologies).

\section{Collection and RNA extraction from peripheral blood mononuclear cells (PBMCs)}

Whole blood was collected from individuals (Princess Alexandra Hospital REC approval 2005/221). Informed consent was obtained from all subjects. PMBCs were purified by a ficoll gradient and RNA extracted by TRIzol. Purified RNA was prepared for Capture as per cell line RNA.

\section{K562 Capture for ERCC spike-in analysis}

ERCC RNA Spike-In Control mix 1 (Life technologies) were spiked into 3 biological replicates of $\mathrm{K} 562$ cells and sequencing libraries created using the TruSeq ${ }^{\circledR}$ Stranded mRNA Sample Preparation Kit (Illumina) with library preparation, precapture-LMPCR, capture hybridization and post-capture LMPCR all carried out as described (Mercer et al, submitted). The ERCC controls present in the 3 biological replicates samples were captured in two technical replicate hybridizations. The two technical replicate captures along with matched precapture K562 sequencing libraries were each sequenced on one lane of an Illumnia ${ }^{\circledR}$ HiSeq.

\section{Capture for ERCC differential transcript abundance analysis}

To investigate differential expression, a second capture-set was performed. ERCC control mixes were spiked into RNA from PBMCs from 9 human individuals (5 with ERCC control mix 1 and 4 with ERCC control mix 2). ERCC mixes 1 and 2 contain the same 92 RNA control transcripts, with $25 \%$ offset by 4 fold, 2 fold and 1.5 fold between the two mixes respectively, while the remaining $25 \%$ are at the same concentration. ERCC controls were spiked in with a final concentration of $1.11 \%$ (1/90 dilution factor). Capture was performed as described ${ }^{5}$. Briefly, libraries were constructed with the TruSeq ${ }^{\circledR}$ Stranded mRNA Sample Preparation Kit (Illumina) and 10 cycles of precapture-LMPCR. At this stage a proportion of each library was retained for precapture control sequencing on an Illumnia ${ }^{\circledR}$ HiSeq. The remaining 
libraries for capture were randomly assigned to one of two multiplex capture pools and two capture hybridizations conducted with $1 \mathrm{\mu g}$ of multiplex library. Captured multiplex samples were amplified with 17 cycles of postcapture-LMPCR. The two multiplex capture samples were pooled and sequenced on one lane of an Illumnia ${ }^{\circledR}$ HiSeq.

\section{Design of Human IncRNA capture oligonucleotides}

Oligonucleotide probe target regions were designed to capture all human long noncoding RNAs using a Roche/Nimblegen SeqCap EZ Choice XL Library. Target regions included all known IncRNA exons from GENCODE $v 12^{11}$, the human lincRNA catalog $^{10}$ and $\operatorname{lncRNAdb}{ }^{13}$. A number of controls were added to the design including 1000 sequences targeting random IncRNA intronic regions (filtered to ensure targeted sequences were $>200 \mathrm{nt}$ and didn't overlap exons of GENCODE $\mathrm{v} 12^{11}$, IncRNAdb ${ }^{13}$, lincRNA catalog ${ }^{10}$ lincRNAs or TUCPs and UCSC mRNAs or ESTs ${ }^{21}$ (downloaded 12.06.2012)), 166 regions $1.1 \mathrm{~kb}$-100bp upstream from putative single exon IncRNAs (filtered to ensure target sequences were $<50 \%$ repetitive and didn't overlap exons or introns of GENCODE v12, IncRNAdb, lincRNA catalog lincRNAs or TUCPs and UCSC mRNAs or ESTs (downloaded 12.06.2012), a 400kb gene desert on human chromosome 7, 44 exons from 14 protein coding genes, a $100 \mathrm{~kb}$ region of E.coli K12 genomic sequence and the ERCC RNA Spike-In Control set (Life technologies). Novel putative coding exons identified by Lindblad-Toh et al. $(2011)^{17}$ were also targeted to investigate their possible connection to known or novel genes. The 3788 candidate exons where filtered to remove any that overlapped GENCODE $\mathrm{v} 12^{12}$ or RefSeq ${ }^{22}$ exons on either strand leaving 2106 exons for targeting. In total the design targeted $\sim 26.5 \mathrm{Mb}$ of hg19 including 29127 IncRNA transcripts from 16453 loci.

Final oligonucleotide probe design and synthesis was performed by Roche/NimbleGen. Probes were allowed to have a maximum of five matches to the genome (as defined by SSAHA ${ }^{23}$ and proprietary bioinformatics) and the oligonucleotide probes synthesized by Roche/Nimblegen covered $85.2 \%$ of target regions directly with an estimated $93.6 \%$ of target regions able to be captured. Capture design co-ordinates (hg19) are provided in Supplementary Data 1.

\section{LncRNA Capture in human tissues and cell lines.}

ERCC RNA Spike-In Control mix 1 (Life technologies) was added to ribodepleted RNA from 20 human tissues and 4 cell lines (K562, MCF-7 and SK-N-SH and Hela, including two biological replicates of K562) to a final dilution of 1/100. Capture was performed as described ${ }^{5}$. Briefly, libraries were constructed with the TruSeq ${ }^{\circledR}$ Stranded mRNA Sample Preparation Kit (Illumina) and 9-11 cycles of precapture-LMPCR were performed depending on the library. Multiplex library pools were created by combining equal amounts of pre-capture libraries from five different tissues, or the five cell line samples and capture hybridization performed with $1 \mu \mathrm{g}$ of each multiplex library pool. Captured multiplex samples were amplified with 17 cycles of postcapture-LMPCR and each multiplex capture of 5 samples was sequenced on one lane of an Illumnia ${ }^{\circledR}$ HiSeq 


\section{Enrichment qPCR}

Successful capture was confirmed by qPCR for the enrichment of both Nimblegen capture controls and capture design specific transcripts using Sybr Green PCR Master Mix and real time cyclers (Applied Biosystems), via the method described previously ${ }^{5}$. Transcripts not specifically captured during the hybridization were also tested via qPCR as a measure of capture specificity. All primers were efficiency tested. Primer sequences are available in Table S1. Any capture hybridizations with qPCR results showing poor enrichment were repeated.

\section{ERCC qPCR}

Primers were designed to ERCC controls spanning a concentration range of $2.5 \times 10^{\wedge}{ }^{5}$ (second lowest to third highest ERCC concentrations) each differing in concentration by 8 fold or less. Primer efficiency of each amplicon was tested by qPCR on a dilution series of purified target DNA and only primer amplicons with an efficiency of $>1.85$ were utilized further. Primer sequences are available in Table S1.

The range and accuracy of qPCR for ERCC quantification was tested on two different sample types in biological replicate. Two biological replicate K562 precapture LMPCR libraries with ERCC spiked in at 1\%, which were also used for CaptureSeq, were utilized for qPCR using $5 \mathrm{ng}$ of cDNA library per well. ERCC standards were also added to $1 \mu \mathrm{g}$ of K562 RNA from the same biological replicates the libraries were made from, keeping the ERCC:RNA ratio the same as used in the library preps. RTPCR was conducted with the SuperScript ${ }^{\mathrm{TM}}$ III cDNA synthesis kit (Life Technologies) using random hexamers.

Quantitative-PCR was performed using Sybr Green PCR Master Mix and real time cyclers (Applied Biosystems) with standard curves for each ERCC present in each plate to allow the absolute quantification of ERCC amount. The two lowest concentration ERCC standards tested (ERCC 00083 and ERCC 00142) could not be consistently placed on a standard curve and hence were at or below the quantitative limit of the qPCR.

\section{Sequencing and Assembly}

Before mapping FastQC was utilized to confirm sequence quality. Reads were trimmed with the Trim Galore Cutadapt ${ }^{24}$ wrapper using the -paired option.

For K562 and PBMC ERCC capture, sequenced reads (.fastq files) were aligned to the human genome using Tophat $2^{25}$ with the firststrand library type option selected. A human Bowtie ${ }^{26}$ index containing the ERCC controls was utilized for Tophat2 mapping, along with a transcriptome index. PBMC pre-capture samples were downsampled to have the same number of mapped reads as their matched capture sample using SAMtools ${ }^{27}$.

For IncRNA capture from tissues and cell lines, sequenced reads (.fastq files) were aligned to the human genome using STAR ${ }^{28}$. A reference annotation from GENCODE $\mathrm{v} 19^{12}$ was supplied during alignment. A splice junction library file was then derived from alignments and used for a second-round alignment solely using TopHat $2^{25}$.

To reduce memory requirements and improve runtimes the IncRNA cell line capture samples were preprocessed with trinity's read normalization ${ }^{29}$ release 2013-02-25 with parameter --max_cov 30. All the reads were mapped to the human genome 
(GRCh37, ENSEMBL 74) considering GENCODE v19 reference annotations using STAR ${ }^{28}$ version 2.3.1z with parameters --outFilterIntronMotifs RemoveNoncanonical -outFilterType BySJout. The resulting sam files were processed to remove the softclipping. Transcriptome assemblies were generated using cufflinks ${ }^{30}$ version 2.1.1 masking rRNA genes and providing the parameters - $u$--library-type fr-firststrand $-b$. The comprehensive transcriptome assembly was generated merging individual assemblies with cuffmerge version 2.1.1.

ERCC read counts were calculated for each mapped library and RPKM values calculated. Mapped read pair counts were calculated with HTseq-count ${ }^{31}$. Average per-basepair read coverage was calculated with BEDTools ${ }^{32}$.

\section{Filtering captured transcripts to create a IncRNA catalogue}

Full length transcript sequences were filtered to retain only those which overlapped tiled capture probe regions annotated as either exons of IncRNAs or the gene desert control. Transcripts with no expression in any cell lines or tissue, which were under 200nt, as well as single exon transcripts with poor strand specificity were then removed. Unreliable transcripts with noncanonical splice junctions were filtered out by removing: transcripts with a non-canonical splice junction that would have been canonical in the antisense orientation; transcripts with almost identical intron chains to other isoforms but which contained a non-canonical splice junction.

Previous gene annotations were employed to perform a comparative analysis with captured transcripts. We utilized Human RefSeq ${ }^{22}$ and UCSC Known Genes ${ }^{21}$ (both downloaded from the UCSC Genome Browser on 07.08.14), GENCODE v19 comprehensive $^{12}$, lincRNAs ${ }^{10}$, TUCPs $^{10}$ and IncRNAdb ${ }^{13}$. Comparative analysis, including the identification of novel exons, introns and transcripts, was performed using CuffCompare (version 2.1.1) 3 $^{33}$. After removing low confidence transcript classes there were 54972 captured transcripts remaining, which were analyzed using CPAT (V1.2.1) ${ }^{14}$ to assign coding potential using the human (hg19) assembly. Some validated IncRNA transcripts have previously been found to be classified as protein coding by genome-wide classification techniques ${ }^{10}$. To alleviate this, we generated a rescue set of transcripts from known IncRNA loci in IncRNAdb ${ }^{13}$ and reclassified these as noncoding. Assembled transcript co-ordinates (hg19), as well as isoform FPKM values, are provided in Supplementary Data 2-7.

To identify and map putative open-reading frames we employed TransDecoder (http://transdecoder.sourceforge.net/) using default parameters. TransDecoder identifies extended open-reading frames and annotates ORFs within human reference genome relative to assemblies, and identified protein sequences with significant homology matches to Pfam-AB database ${ }^{15}$.

To identify novel putative protein-coding transcripts from loci previously classified as lincRNAs, we initially filtered for all assembled transcripts classified as "known coding" or "novel coding" coding that showed no overlap to GENCODE v19 coding transcripts. This identified 2454 transcripts from 939 loci. We further filtered these transcripts to omit any with overlap to pseudogenes (Pseudogene Annotation Set from GENCODE v19) and other coding exons (from UCSC Known Genes and RefSeq genes). We required each transcript to encompass a full length open reading frame 
and were queried to the $\mathrm{Pfam}^{15}$ database to ascertain significant homology to known protein domains.

\section{Captured transcripts from putative novel coding exons}

Transcripts overlapping putative novel coding exons from identified and filtered using a similar procedure to the IncRNA catalogue. Probed exons were filtered to remove any annotated as protein coding with an updated dataset (GENCODE v19 comprehensive). Assembled transcripts that overlapped a tiled captured probe in a target region were filtered to retain those captured solely by probes to putative coding exons. Transcripts with no expression in any cell lines or tissue, which were under 200nt or which contained a non-canonical splice junction were removed. CuffCompare (version 2.1.1) was utilized to assess transcript novelty and classification and reduce redundancy. To improve classification accuracy, transcripts overlapping putative novel coding exons were combined with the 54972 transcripts from the IncRNA capture and CuffCompare run using the same reference set as previous. After removing low confidence transcript classes 831 transcripts captured solely by probes to putative novel coding exons remained. Some transcripts were targeted by probes to IncRNA exons and putative novel coding exons and were amongst the 54972 transcripts that passed IncRNA catalogue filtering. Combining these two sets gave 1042 transcripts with a strand specific overlap to the 2060 putative novel coding exons. CPAT $(\mathrm{V} 1.2 .1)^{14}$ was used to help assign coding potential to each transcript using the human (hg19) assembly and potential ORFs mapped using TransDecoder (as above).

Completely novel transcripts with no overlap to any GENCODE v19 annotations and multiple putative novel coding exons as well as GENCODE v19 lincRNAs with one or more putative novel coding exons were further investigated manually to ascertain their coding potential.

The classification of novel exons as $5^{\prime}$, internal, or $3^{\prime}$ was determined from their position in the assembled capture transcripts. To prevent double counting (as one novel exon could be internal to one transcript and a terminal exon at another) a classification hierarchy of internal-5'-3' was used. Exon classifications are provided in Table S2.

Coordinates of assembled transcripts and expressed putative novel coding exons (hg19), as well as isoform FPKM values, are provided in Supplementary Data 7-9. A list of all assembled transcripts and their associated putative novel coding exons and GENCODE genes are provided in Table S2.

\section{Bioinformatics}

In addition, to the above bioinformatic analysis, a number of tools were utilized during general data analysis, including BEDTools ${ }^{32}$, SAMtools ${ }^{27}$ Kent Source Tools and internal unix/perl/python scripts. Statistical analysis and graphing was performed with GraphPad Prism and R. Nucleotide logos were generated with WebLogo ${ }^{34}$.

\section{Sample Clustering and differential gene expression analysis}

Output of HTseq-count was used an input into DESeq $2^{35}$ and/or edge ${ }^{36}$. Data was prepared for clustering by variance stabilizing transformation in DESeq2. Clusters 
were visualized using the heatmap. 2 function of the gplots program in R. Proportion of alternatively spliced exons was determined by measuring the number of exons entirely overlapped by an introns using OverlapSelect utility.

\section{Saturation analysis}

The saturation of ERCC controls was tested by weighted non-linear regression. A straight line fit was compared to segmental linear regression using Akaike's information criterion, which identified segmental linear regression as the best fit for CaptureSeq experiments, while a straight line fit was generally preferred for RNAseq. Akaike's information criterion was then utilized to determine the best break point for segmental linear regression.

\section{Variability in measurement of ERCC abundance}

Variability in the measurement of ERCC standard abundance was calculated as the FPKM standard deviation for 3 biological replicates divided by the mean FPKM. This measure removes the impact of expression level and provides a value for how consistent the FPKM value was between each biological replicate. A ratio of this value between RNAseq and CaptureSeq was calculated and graphed. Values above one show RNAseq is more variable, values below -1 show CaptureSeq is more variable.

\section{CaptureSeq performance against ERCC variables.}

The impact of differences in ERCC GC\% and length was determined by calculating the average enrichment of each ERCC probe for each CaptureSeq technical replicate. A significant relationship was observed between ERCC length and enrichment, but not for GC\%. To help remove the influence of ERCC expression abundance, non-linear regression was utilized to fit a curve (2-phase decay) to the enrichment values, as determined by Akaike's information criterion. The enrichment residuals were then plotted against ERCC length and GC\%.

\section{Conservation Analysis}

Human nucleotide conservation score, was retrieved from UCSC Genome Browser (PhyloP Basewise Conservation with 100 Vertebrate MultiZ Alignment ${ }^{37,}{ }^{38}$. This evaluates individual nucleotides for both accelerated (faster than expected under neutral drift) and conserved (slower than expected evolution). Conservation scores represent - $\log p$-values under a null hypothesis of neutral evolution.

To provide a profile of conservation across exons, we download the 100 Vertebrate Conservation wiggle track (phyloP100All) from UCSC Genome Browser. 'Pile-up' histograms were generated from average coverage of conservation scores across window centered on exon 3' splice site.

\section{Disease SNPs}

We employed the following datasets to determine overlap between exons and human variation. Coordinates for common simple nucleotide polymorphisms (dbSNP 137) that are found in greater than $1 \%$ of the humans ${ }^{39}$, we downloaded from NCBI. Cancer associated SNPs were downloaded from the Catalogue Of Somatic Mutations In Cancer database of somatic mutations found in human cancer ${ }^{40}$. Mutations and 
SNPs associated with disease were download from the public Human Gene Mutation Database (HGMD) that show the genomic coordinates of disease-associated variants in the public version of the database ${ }^{41}$.

\section{ONLINE METHODS REFERENCES}

21. Hsu, F. et al. Bioinformatics 22, 1036-1046 (2006).

22. Pruitt, K.D. et al. Nucleic Acids Res 42, D756-763 (2014).

23. Ning, Z., Cox, A.J. \& Mullikin, J.C. Genome Res 11, 1725-1729 (2001).

24. Martin, J.A. \& Wang, Z. Nat Rev Genet 12, 671-682 (2011).

25. Kim, D. et al. Genome Biol 14, R36 (2013).

26. Langmead, B. \& Salzberg, S.L. Nat Methods 9, 357-359 (2012).

27. Li, H. et al. Bioinformatics 25, 2078-2079 (2009).

28. Dobin, A. et al. Bioinformatics 29, 15-21 (2013).

29. Haas, B.J. et al. Nat Protoc 8, 1494-1512 (2013).

30. Trapnell, C. et al. Nat Protoc 7, 562-578 (2012).

31. Anders, S., Pyl, P.T. \& Huber, W. bioRxiv (2014).

32. Quinlan, A.R. \& Hall, I.M. Bioinformatics 26, 841-842 (2010).

33. Trapnell, C. et al. Nat Biotechnol 28, 511-515 (2010).

34. Crooks, G.E., Hon, G., Chandonia, J.M. \& Brenner, S.E. Genome Res 14, 11881190 (2004).

35. Love, M.I., Huber, W. \& Anders, S. bioRxiv (2014).

36. Robinson, M.D., McCarthy, D.J. \& Smyth, G.K. Bioinformatics 26, 139-140 (2010).

37. Blanchette, M. et al. Genome Res 14, 708-715 (2004).

38. Pollard, K.S., Hubisz, M.J., Rosenbloom, K.R. \& Siepel, A. Genome Res 20, 110121 (2010).

39. Sherry, S.T. et al. Nucleic Acids Res 29, 308-311 (2001).

40. Forbes, S.A. et al. Nucleic Acids Res 39, D945-950 (2011).

41. Stenson, P.D. et al. Genome Med 1, 13 (2009). 

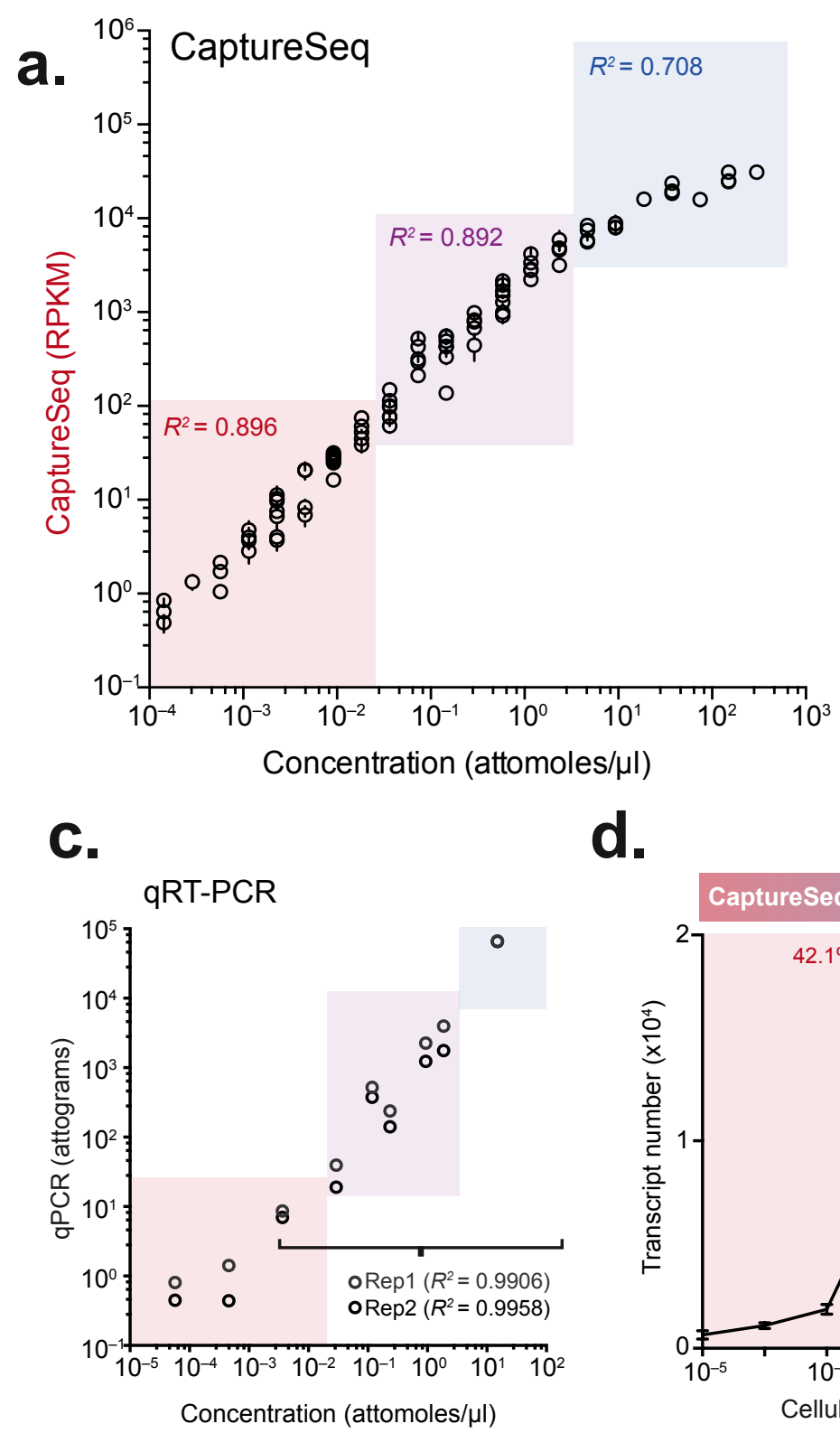

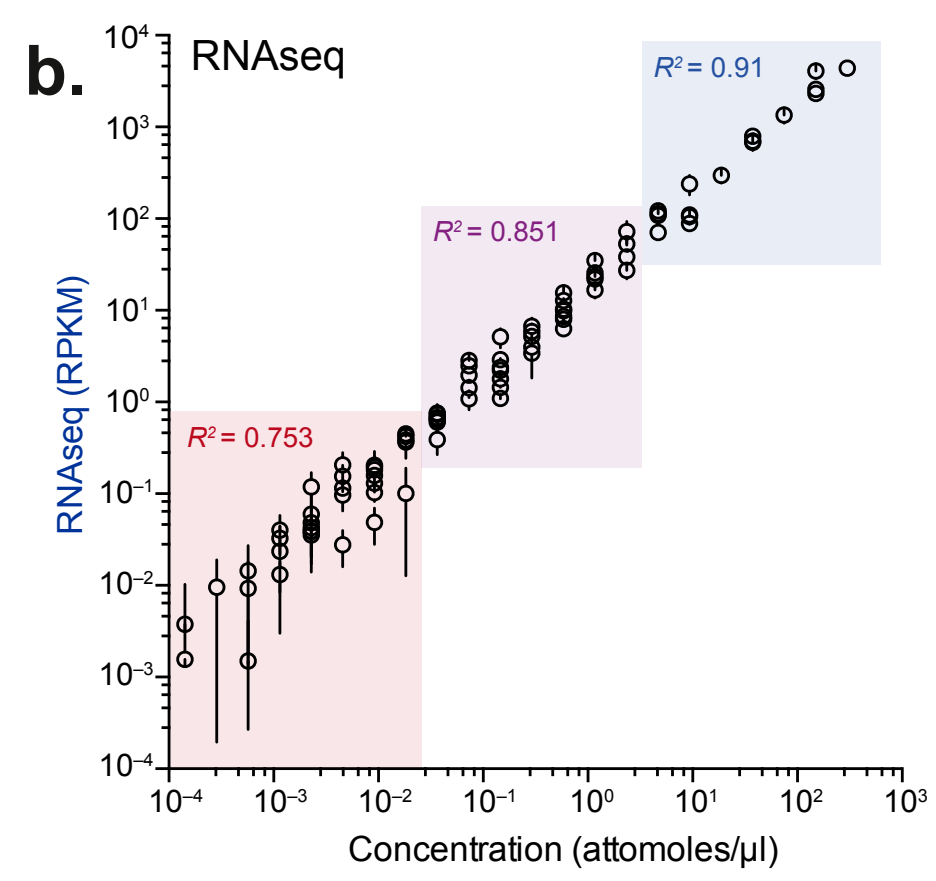

e.
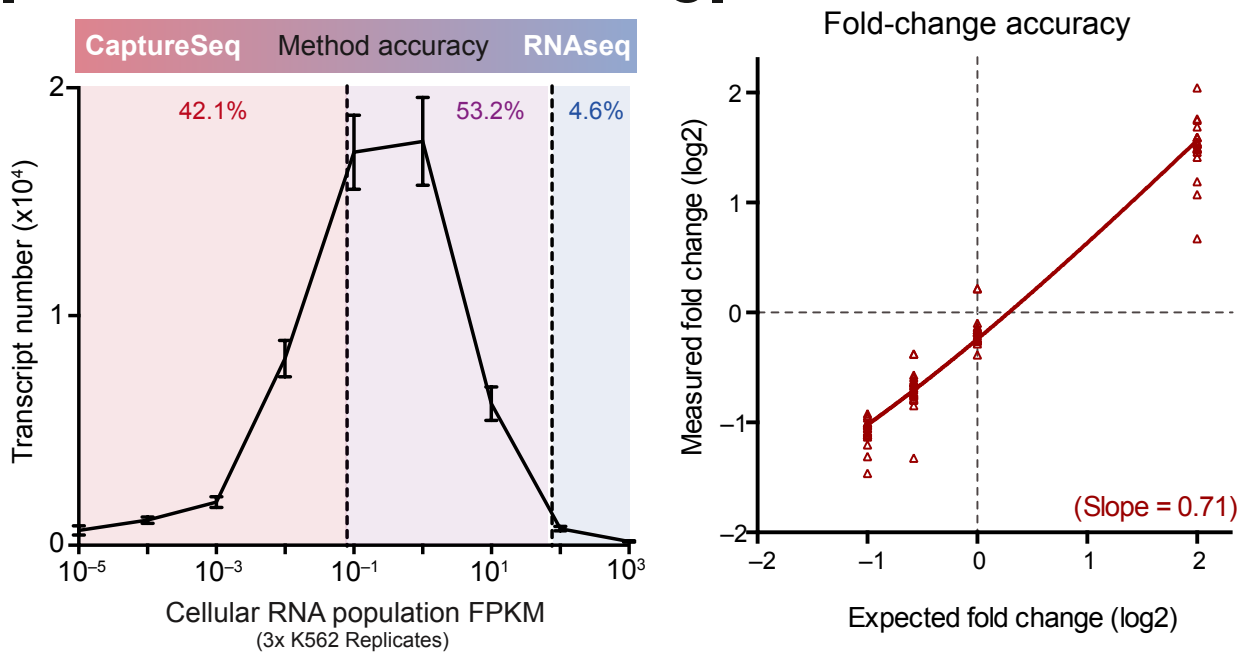
a. CaptureSeq for gene profiling:
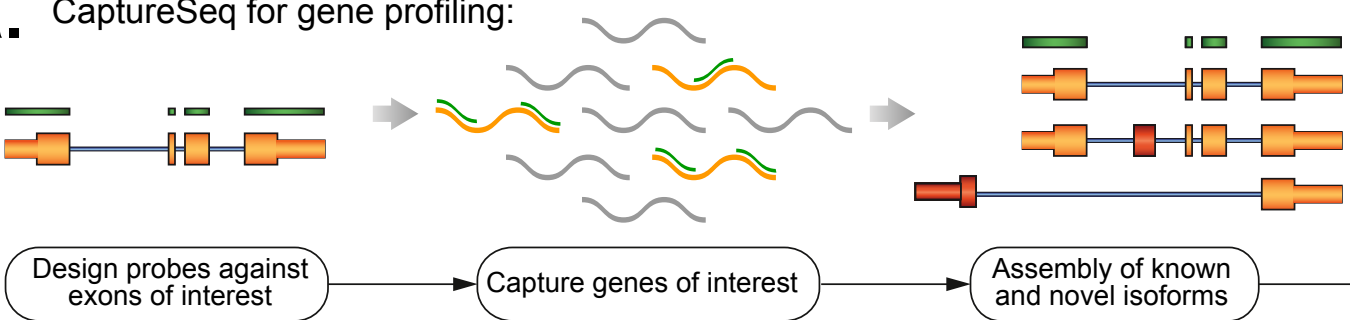

Assembly of known
and novel isoforms

Profiling of expression and splicing

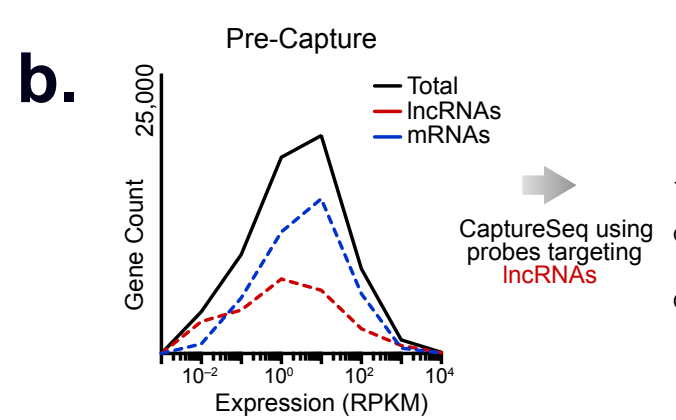

Enrichment for IncRNAs

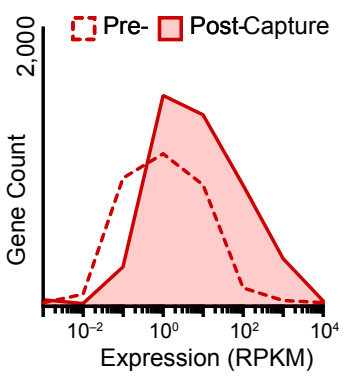

Depletion of mRNAs

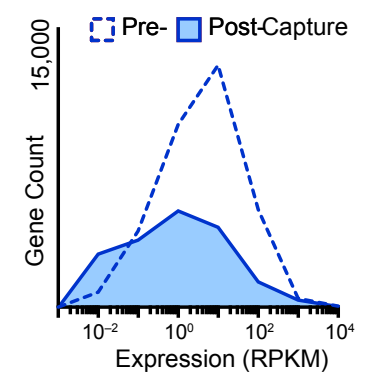

c.

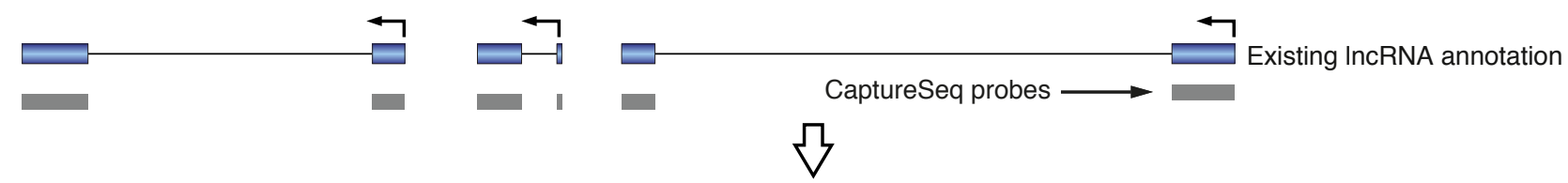

CaptureSeq merges IncRNAs

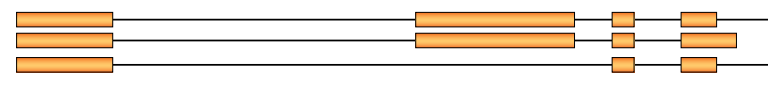

FANTOM 5 TSS peaks (Permissive) i

Fantom 5 Enhancer

chr1 23,875,400

$2 \mathrm{~kb}$

| IIII III

(1) (2) $\mathrm{H}+\mathrm{HWH}-\mathrm{H} \mid \mathrm{H}-\mathrm{|}$
(3)

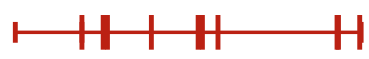

CaptureSeq probes
(4)

HII

そ
(5)

\section{d.}

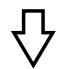

CaptureSeq merges 3 coding genes and 2 IncRNAs gene into a single 3,188 aa gene

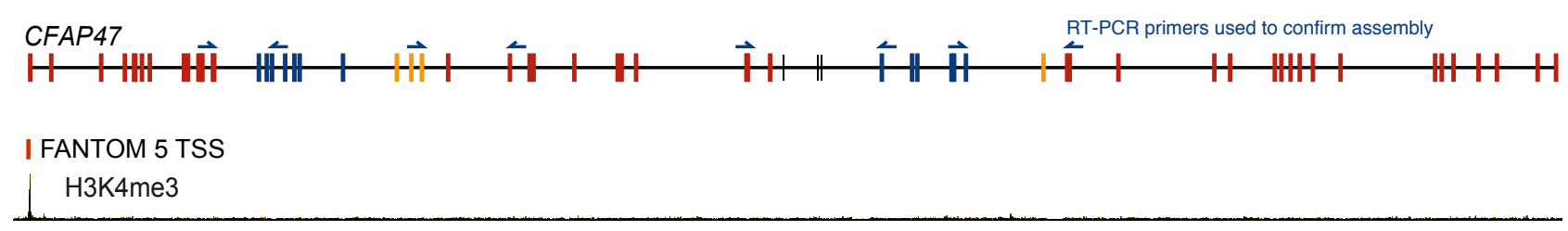

\section{Vertebrate conservation}




\section{SUPPLEMENTARY RESULTS}

\section{Design validation with genomic DNA controls}

We performed an initial genomic DNA capture to confirm the efficacy of the IncRNA capture design. We found that $74.5 \%$ of gDNA sequenced reads overlapped probes, with $96.5 \%$ of probes having more than 1-fold coverage across their entire length (Figure S7d).

The IncRNA design included probes targeting intergenic regions to measure potential DNA contamination. By comparing the relative enrichment of reads aligning to experimental probes (that target exons) to the control probes can provide an indication as to the existence of DNA contamination. We found a $\sim 1,874$ fold relative enrichment of experimental probes targeting exons relative to control probes when capture is performed with cDNA libraries. By comparison, we observe a 1.57-fold enrichment for reads aligning to control probes relative to experimental probes when capture is performed with genomic DNA. Taken together these results demonstrate a very low level of potential DNA contamination that could affect our IncRNA capture results.

\section{Read Coverage across transcripts is similar between RNAseq and CaptureSeq}

The single exon nature of ERCC standards makes them well suited for the analysis of read coverage heterogeneity. Both RNAseq and CaptureSeq exhibit similar and highly reproducible sequence coverage across the full length of standards, with only minor (7.8\%) divergence observed (Figure S4c). In those regions of ERCC transcripts that exhibit different coverage, we observe no significant association with nucleotide content (paired t-test, $p=0.86$; Figure $\mathbf{S 4 d} \mathrm{d}$ ). We also examined read coverage across annotated control genes that were included within probe designs. We observed similar and highly reproducible sequence coverage across the full length of control genes in both RNAseq and CaptureSeq (Figure S4e). This collectively suggests that sequence-specific biases in RNAseq library preparation, sequencing and alignment introduce greater technical variation than CaptureSeq specific protocol steps of hybridization and washing.

\section{Potential biases for CaptureSeq enrichment}

For moderately abundant ERCC standards (0.0366-2.34 attomoles/ $\mu$ l) we compared the quantification of ERCC standards relative to their length and GC\%. For CaptureSeq, we observe an inverse relationship between standard length and enrichment $p<0.001$ (Figure S4a). Given that short sequences are generally underrepresented in RNAseq libraries ${ }^{1}$, this suggests CaptureSeq may perform better in the detection and quantification of short transcripts. We observe no significant relationship between enrichment and average transcript GC\% (Figure S4b), though this may not hold true at GC percentages not covered by the ERCC standards.

\section{Duplication bias}

We considered whether PCR amplification artifacts may be introduced by the additional PCR step in the CaptureSeq. We previously found little divergence in 
sequence read coverage across genes between RNAseq and CaptureSeq, and no evidence of 'block' coverage characteristic of PCR duplication (Figure S4e).

Given the higher coverage of exons by reads in CaptureSeq, duplicated reads likely comprise both PCR duplication and bona-fide identical sequenced reads. We assessed the quantitative accuracy for measuring ERCC standards following the removal of duplicate reads, finding a slightly lower correlation following duplicate removal ( $R^{2}-0.9966$ to 0.9989$)$ that is exacerbated for abundant ERCC standards. Therefore, we recommend not removing duplicate reads, however, digital PCR methods ${ }^{2}$ will be required to distinguish relative contributions of PCR duplication biases and bona-fide identical sequenced reads.

\section{Linear quantitation of RNAseq and CaptureSeq}

In addition to the correlations observed between the measured and known abundance of ERCC standards, we also examined the linearity of quantitation (how increases in ERCC abundance equate to a proportional increase in measured expression) for RNAseq and CaptureSeq by non-linear regression with a straight line fit. As previously reported, RNAseq shows a linear quantitation over six orders of magnitude $^{1}$. CaptureSeq displayed regression slopes of $0.050(95 \% \mathrm{Cl} 0.0464$ to $0.05334)$ for technical replicate one and $0.033(95 \% \mathrm{Cl} 0.03063$ to 0.03581 ) for technical replicate two (each technical replicate capture hybridization contained three biological replicate RNA samples, see Online Methods).. These lower slopes were largely due to CaptureSeq saturation at high expression levels; below the saturation cutoff in the CaptureSeq dynamic range the linear slopes were 0.7832 (95\% $\mathrm{Cl} 0.7391$ to 0.8273$)$ and $0.6358(95 \% \mathrm{Cl} 0.6007$ to 0.6710$)$ respectively. Given that CaptureSeq provided a linear response over $\sim 4$ orders of magnitude (and possibly more as the limit of the dynamic range was below the lowest ERCC concentration), the quantitative efficiency with which CaptureSeq detected a 10 fold change in transcript abundance below the saturation cutoff was $>=90 \%$.

At low expression levels the linear quantitation of RNAseq was above $1(1.2495 \% \mathrm{Cl}$ 1.006 to 1.474), demonstrating the change in measured expression was larger than the actual change in ERCC abundance. This is due to the paucity of detected reads for many low level ERCC standards, including a number that were not detected in individual replicates. This results in artificially low RPKM values for many of the rarest ERCC standards, maintaining the appearance of dynamic range at these concentrations. However, the lower correlation values and high variability between replicates (Figure1, S2, S3) make it clear these values are not accurate quantitative measurements of transcript abundance.

Measuring the fold differences in gene expression between samples with Capture Sequencing

We utilized a second set of Capture samples to examine the potential for CaptureSeq to identify gene expression differences between groups of samples. RNA from 9 individuals was spiked with either the previously utilized ERCC mix number 1 (5 samples) or ERCC mix number 2 (4 samples). The ERCC controls in mix 1 and 2 differ by known amounts allowing the determination of both true and false positive and negative calls for differential transcript abundance. The samples used were randomized to one of two multiplex capture hybridizations. 
The correlations between the expression levels of each ERCC control were very high (average mix 1 Pearson $R^{2}=0.968$, average mix 2 Pearson $R^{2}=0.970$ ), with representative comparisons shown in Figure S5a. Similar results were observed with sample clustering (Figure S5b). These results confirm samples from different multiplex captures have very high replicability with only minor effects from the capture process (hybridization, washing, post capture LMPCR). Nevertheless, we recommend randomising samples to different captures as this will ensure the true (although small) technical variability of the procedure is represented in the sample results.

Similar to Figure $\mathbf{1}$ and S2, we investigated the CaptureSeq dynamic range and saturation point for the 9 samples. Despite a lower overall sequencing depth (average of $\sim 8.4$ fold less ERCC reads per sample), all ERCC controls were consistently detected Figure S5c-e. Segmental linear regression supported a linear slope up to a saturation point of 1.3 and 0.976 attomoles/ $\mu$ for mix 1 and mix 2 respectively, similar to the 2.34 attomoles/ $\mu$ l saturation point identified for the first set of samples. Hence CaptureSeq provides a linear response over $\sim 4$ orders of magnitude and possibly more because the limit of the dynamic range was below the lowest ERCC concentration. Linear slopes (out of 1 ) prior to saturation of 0.45 (mix 1 ) and 0.35 ( $\operatorname{mix} 2$ ) suggest the quantitative efficiency with which CaptureSeq detected a 10 fold change in transcript abundance was around or over $80 \%$.

We next examined the performance of CaptureSeq in measuring the differences in transcript abundance between ERCC mixes. Although CaptureSeq performed well in detecting RNA abundance differences, the detected fold changes and linear slope suggest CaptureSeq can underestimate the abundance differences between samples (Figure 1e, S6a). Notably, no relationship was found between ERCC concentration and the accuracy of fold change measurement, i.e.: the differences in ERCC abundance between the mixes were measured just as accurately above the saturation threshold as below it. An explanation for this observation is that in a capture hybridization containing multiple libraries, each ERCC transcript is competing with the same transcript from the other libraries to bind a capture probe. When the total amount of probe is limiting, the same transcript in each library would compete and bind to the probes in proportion to its abundance and therefore accurate fold change information is obtained even when expression level information is not.

To determine if the detected fold differences were sufficient to identify differential expression (DE) we tested the CaptureSeq results with edge ${ }^{3}$. Under standard conditions with a 1\% FDR, 100\% of the ERCC controls with 4 fold or 2 fold changes were correctly identified as DE, as were $52 \%$ of controls with 1.5 fold changes (Figure S5f). Two (of 23) ERCC controls with no change between the mixes were called DE by edgeR, giving a false positive rate of $9 \%$ and a recall value of 0.84 . Sensitivity was highest for ERCC controls at moderate concentrations, but CaptureSeq also identified DE at the lowest and highest concentrations.

We compared the CaptureSeq results for transcript abundance and differential gene expression profiling to those of standard RNAseq performed on the same libraries prior to capture. Precapture control libraries were sequenced to a greater depth and had, on average, 6.3 times more mapped reads than the CaptureSeq libraries. This allowed us to compare CaptureSeq and RNAseq results with a matched number of 
mapped reads (using downsampling), but also to investigate if RNAseq could match CaptureSeq in identifying DE at low expression levels though deeper sequencing.

Unlike CaptureSeq, matched RNAseq showed no general underestimation of fold change differences (linear slope $0.98,95 \% \mathrm{Cl} 0.81$ to 1.1 , compared to $0.7195 \% \mathrm{Cl}$ 0.66 to 0.75 , Figure S6a). However, matched RNAseq provided no results for 11 lowly expressed ERCC controls and the variability of the measured fold changes was larger as a result of less accurate fold change measurements for many other standards (Figure S6b). Testing for DE, with matched RNAseq and a 1\% FDR, revealed $65 \%$ of the ERCC controls with 4 fold, $57 \%$ of 2 fold and $52 \%$ of 1.5 fold changes were correctly identified as DE. One (of 23) ERCC controls with no change between the mixes was called $\mathrm{DE}$, giving a false positive rate of $4 \%$ and a recall value of 0.58 (Figure S6c). This is a similar false positive, but much lower recall value, than obtained with CaptureSeq due to the decreased ability of RNAseq to identify low level transcripts as DE. Repeating this analysis with all RNAseq reads (6.3 times more mapped reads per library than CaptureSeq) gave the same false positive rate as CaptureSeq, but the recall rate was still lower (albeit improved) at 0.73 (Figure S6d).

This strengths and weaknesses of each technique to identify DE can be visualized by plotting the expression of each differentially expressed standard in comparison to the likelihood, from edgeR, it is DE (Figure S6e). RNAseq shows a clear decrease in its ability to detect DE as expression levels decrease, although (and consistent with RNAseq showing a more linear response to expression level changes), RNAseq appears to perform better at providing high confidence calls when expression level changes are small (see 1.5 fold change results). Collectively, these results demonstrate that CaptureSeq can be used to identify DE over a wide range of gene expression levels and that CaptureSeq provides a more accurate method for quantifying DE of lowly expressed genes, such as IncRNAs. While performing RNAseq at increased read depths does not provide an efficient means of emulating the advantages of CaptureSeq.

\section{Proportion of cellular transcriptome better quantified by CaptureSeq}

To provide a context to the advantages of CaptureSeq, we next compared the concentrations of ERCC standards to the total RNA population from K562 cells. Based on our above analysis, we estimate that $42.1 \%$ of transcripts would be better quantified using CaptureSeq (Figure 1d). RNAseq and CaptureSeq would perform comparably well for $53.2 \%$ transcripts, and RNAseq would perform better for the top 4.6\% expressed transcripts that are enriched for housekeeping, structural or metabolic genes. By contrast, the lowly expressed genes in K562 cells for which CaptureSeq provides superior quantitative accuracy are enriched for transcription factors, and genes causally associated with cancer (CancerCensus) and human disease (OMIM, HGMD and BIOBASE) (Figure S7a-c). Although these estimates may vary in other cell-types or tissues according to transcriptome size and complexity, they nevertheless demonstrates the scope of genes for which CaptureSeq may be advantageous. 


\section{Identification of novel coding exons}

A large-scale study of mammalian conserved elements previously predicted $\sim 3800$ novel protein-coding exons in human ${ }^{4}$. Of these, 2060 exons had no prior evidence of expression. To determine if these putative coding exons create novel isoforms and/or completely novel coding genes, we examined their expression simultaneously with IncRNAs across 20 human tissues and 4 cell lines by CaptureSeq.

We found $543(26 \%)$ of the putative novel coding exons were expressed in at least one tissue. Most exons (457 or $84.2 \%$ ) were incorporated into transcripts that overlapped 333 GENCODE gene annotations (208 coding genes, 72 pseudogenes and 29 lincRNAs). This reveals that only a minority of the expressed novel exons could potentially form completely novel coding genes, with most instead forming novel exons of annotated coding genes or suggesting coding potential in transcripts otherwise thought to be noncoding. The vast majority $(>80 \%)$ of putative coding exons associated with pseudogenes match known pseudogene exons, suggesting these putative novel coding exons predictions could be detecting residual pieces of open read frame.

Focusing our analysis on the 315 novel exons to GENCODE v19 coding genes and IncRNAs (lincRNAs and antisense transcripts). We find 72 new 5', 51 new 3' and 192 new internal exons, with examples of a new $5^{\prime}$ exons correcting transcriptional and translation start sites and novel alternative internal exons (Table S2 Figure S13A-B). A standout example are the 17 novel exons found to connect together several HMCN2 gene fragments on chr 9 to create a 2836 amino acid ORF. This may not be full length, using the existing exons and splice junctions from our CaptureSeq transcripts, ILL_076783 ${ }^{5}$ and ENST00000428715.1 predicts a putative 3703 amino acid ORF (Figure S13C).

Delineating whether transcripts are coding or IncRNAs can be challenging, especially when transcript structures are incomplete. We re-examined 27 GENCODE lincRNA loci with 1 or more putative coding exons, identifying $16 / 27$ as potential coding transcripts, including 4 which contained Pfam $^{6}$ domains (TableS3).

The remaining 86 novel coding exons with no overlap to known genes formed 51 novel transcribed loci. The assembly of putatively coding transcripts without any overlap with GENCODE genes or pseudogenes allows the identification completely novel coding genes, but also has a high probability of identifying false positives. Therefore we selected all novel transcript loci with 2 or more putative coding regions for closer examination. This filtered the novel loci from 51 to 11, 10 of which potentially encode a peptide/protein (TableS3). Due to the limitation in assembling whole transcripts when only capturing a small number of exons 6/10 ORFs were incomplete at the $3^{\prime}$ end and it is plausible four of these form novel extensions to known coding genes. This leaves an estimate of 6 novel coding loci (TableS3, Figure S13D), 3 of which contained Pfam $^{6}$ domains. Probable novel coding loci included ORFs with similarity to mouse fatty acid desaturase 2-like protein and keratins. While we identify possible ORF altering extensions to proteins such as absent in melanoma 1-like (AIM1L) and the poorly annotated protein C4orf50.

These results clearly demonstrate the ability of CaptureSeq to characterise poorly annotated genes, integrating novel exons into existing coding genes as well as identifying novel coding genes missed by previous annotation efforts. 


\section{SUPPLEMENTARY REFERENCES}

1. Jiang, L. et al. Synthetic spike-in standards for RNA-seq experiments. Genome Res 21, 1543-51 (2011).

2. Shiroguchi, K., Jia, T.Z., Sims, P.A. \& Xie, X.S. Digital RNA sequencing minimizes sequence-dependent bias and amplification noise with optimized single-molecule barcodes. Proc Natl Acad Sci U S A 109, 1347-52 (2012).

3. Robinson, M.D., McCarthy, D.J. \& Smyth, G.K. edgeR: a Bioconductor package for differential expression analysis of digital gene expression data. Bioinformatics 26, 139-40 (2010).

4. Lindblad-Toh, K. et al. A high-resolution map of human evolutionary constraint using 29 mammals. Nature 478, 476-82 (2011).

5. Gascoigne, D.K. et al. Pinstripe: a suite of programs for integrating transcriptomic and proteomic datasets identifies novel proteins and improves differentiation of protein-coding and non-coding genes. Bioinformatics 28, 3042-50 (2012).

6. Finn, R.D. et al. Pfam: the protein families database. Nucleic Acids Res 42, D222-30 (2014).

7. Wang, L. et al. CPAT: Coding-Potential Assessment Tool using an alignmentfree logistic regression model. Nucleic Acids Res 41, e74 (2013).

8. Cabili, M.N. et al. Integrative annotation of human large intergenic noncoding RNAs reveals global properties and specific subclasses. Genes Dev 25, 191527 (2011).

9. Harrow, J. et al. GENCODE: the reference human genome annotation for The ENCODE Project. Genome Res 22, 1760-74 (2012).

10. Amaral, P.P., Clark, M.B., Gascoigne, D.K., Dinger, M.E. \& Mattick, J.S. IncRNAdb: a reference database for long noncoding RNAs. Nucleic Acids Res 39, D146-51 (2011).

11. Consortium, F. et al. A promoter-level mammalian expression atlas. Nature 507, 462-70 (2014). 


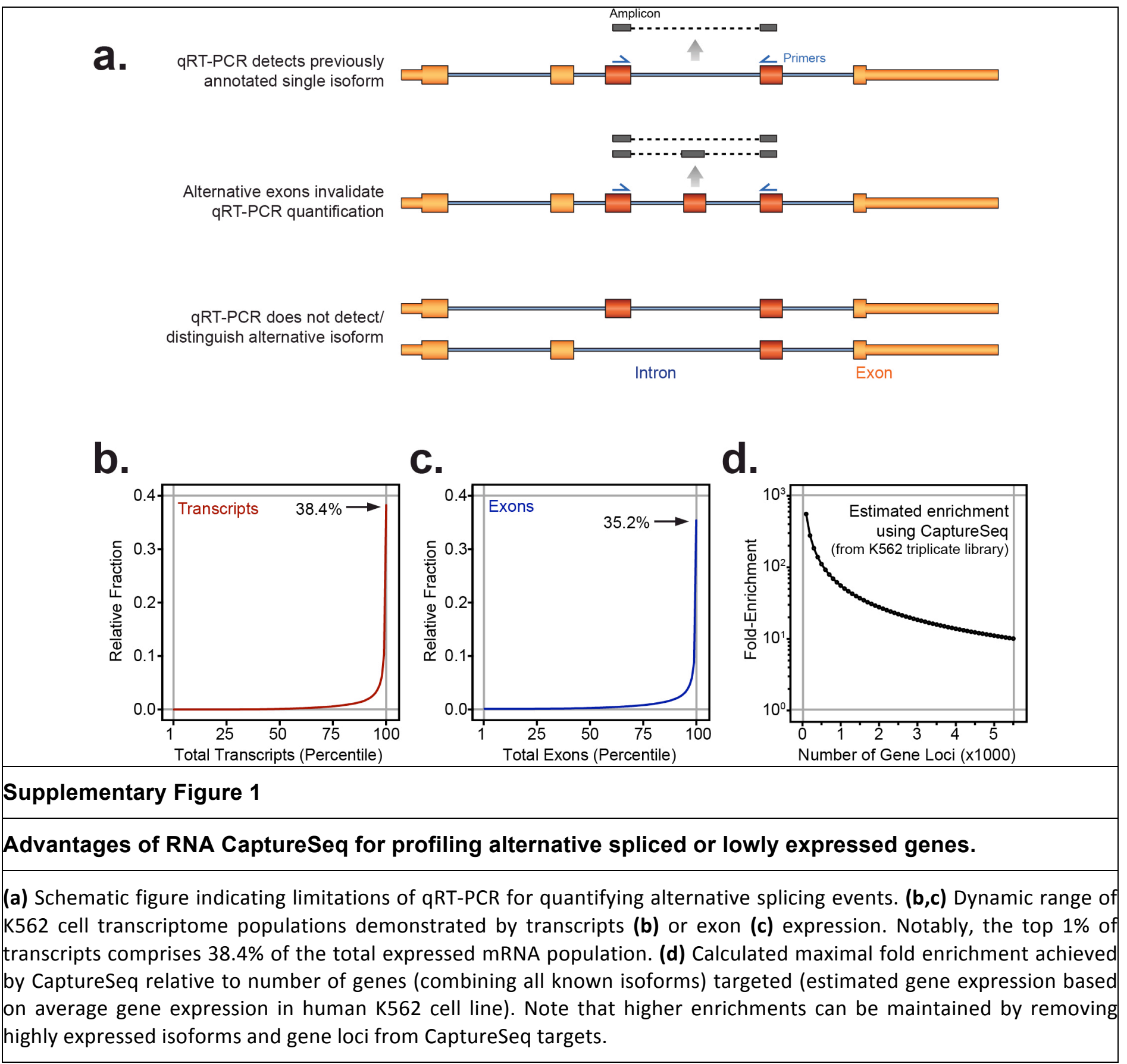



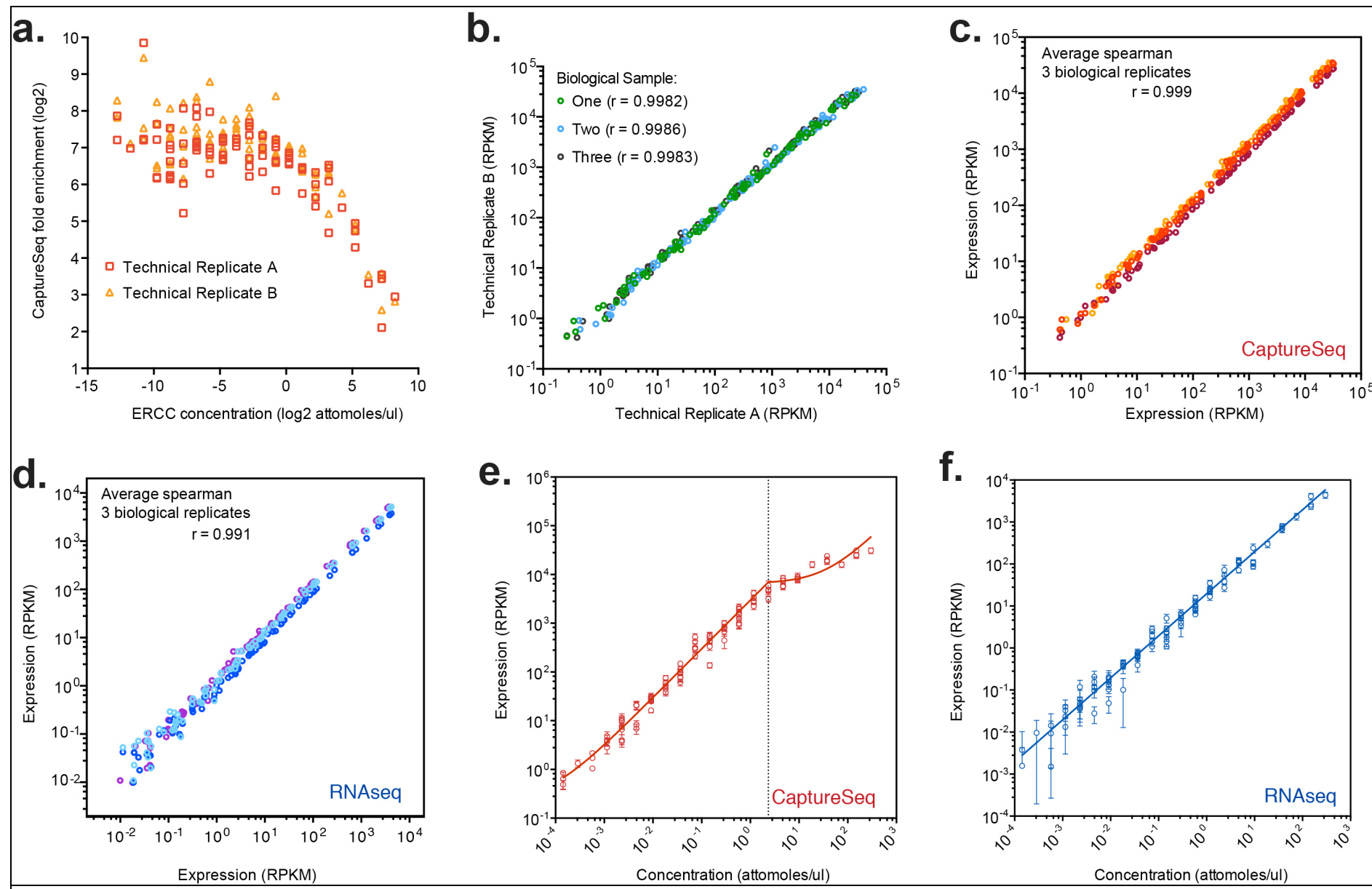

\section{Supplementary Figure 2}

\section{Comparative analysis of ERCC Spike In quantification using RNA sequencing and CaptureSeq}

(a) Fold enrichment achieved by CaptureSeq for each ERCC standard. High and variable enrichment at low ERCC concentrations results from low and sporadic alignment of RNAseq reads to ERCC standards. Decreasing enrichments at high ERCC concentrations is due to CaptureSeq saturation. Each technical replicate capture hybridization contained three biological replicate samples. (b) Spearman correlation of measured abundance by CaptureSeq of ERCC probes for three biological replicate samples in technical replicate. (c,d) Average Spearman correlation of measured abundance of ERCC probes for 3 biological replicates of CaptureSeq (c) and RNAseq (d). (e) Segmented regression analysis indicates inflection point in the measured abundance of ERCC probes by CaptureSeq at an ERCC concentration of 2.34 attomoles/ $\mu$ l (dotted line). $n=3$ biological replicates, error bars are s.d. (f) RNA sequencing exhibits a linear profile across the range of ERCC concentrations it detects. $n=3$, biological replicates, error bars are s.d. 

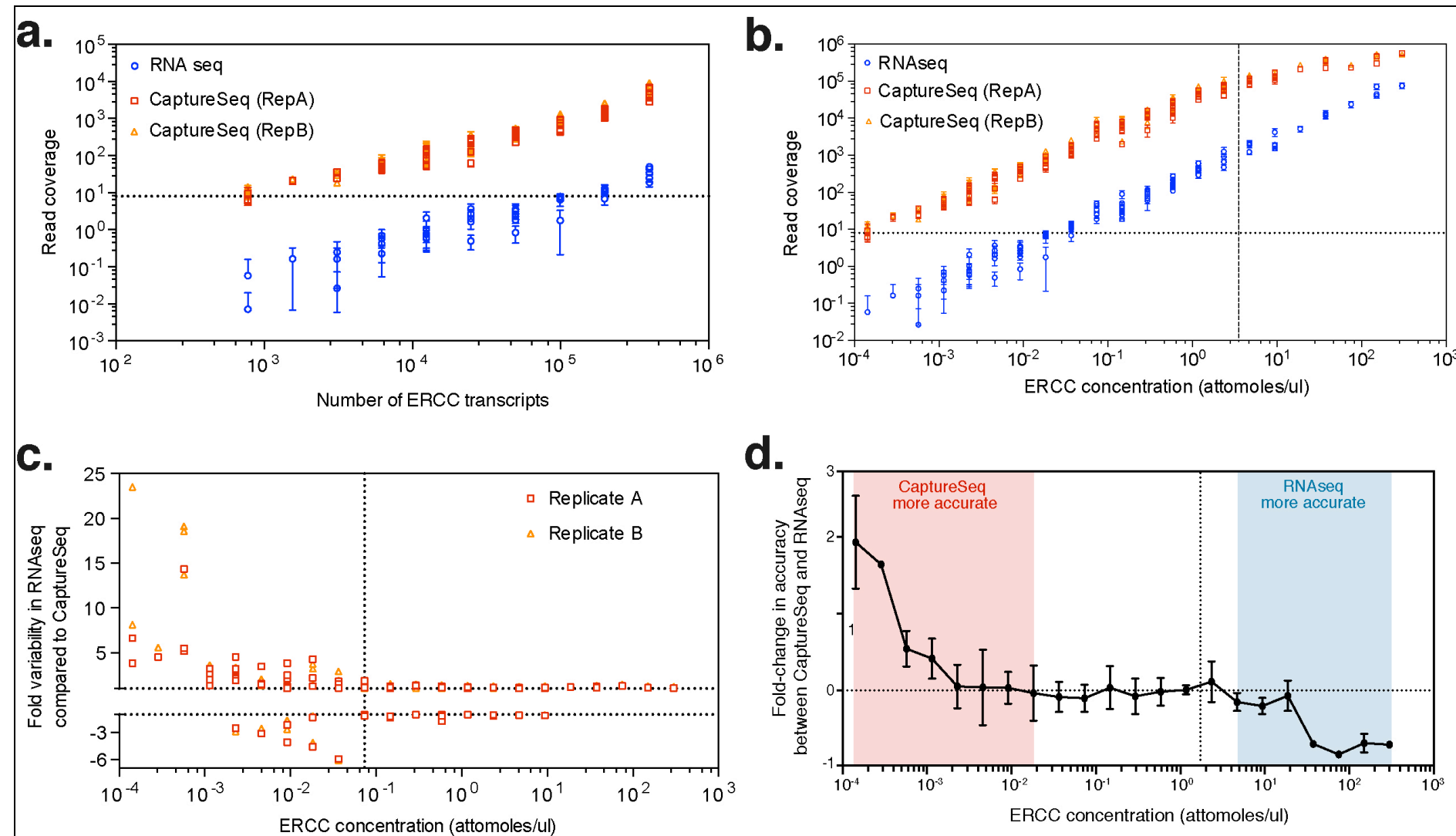

\section{Supplementary Figure 3}

\section{Enrichment in read coverage of ERCC RNA Spike-In by CaptureSeq}

$(\mathbf{a}, \mathbf{b})$ Averaged read coverage for each ERCC probe from RNAseq and CaptureSeq. $n=3$ biological replicates, error bars are s.d. Horizontal dotted line shows 8 fold coverage. RepA and RepB are technical replicate capture hybridizations containing three biological replicate samples. (a) Number of ERCC transcripts required for 8-fold coverage. (b) Concentration of ERCC transcripts required for 8 fold coverage. Vertical dotted line marks concentrations above which CaptureSeq is saturated. Lowest three concentrations of probes $(<0.00114$ attomoles $/ \mu l)$ have zero coverage in more than $50 \%$ of RNAseq libraries. (c) Fold difference in variability between RNAseq measurement of ERCC abundance and CaptureSeq technical replicates ( $n=3$ biological replicates). Horizontal dotted line is at 1 and -1 (no difference in variability). Values above one show RNAseq is more variable, values below -1 show CaptureSeq is more variable. Vertical dotted line is ERCC concentration that allows consistent 8 -fold coverage by RNAseq. RNAseq is more variable at low expression levels. (d) Mean difference between CaptureSeq and RNAseq accuracy in measuring ERCC abundance. Shows RNAseq provides less accurate expression measurements at low levels but is more accurate at high levels $n=3$ biological replicates, error bars are s.d. 

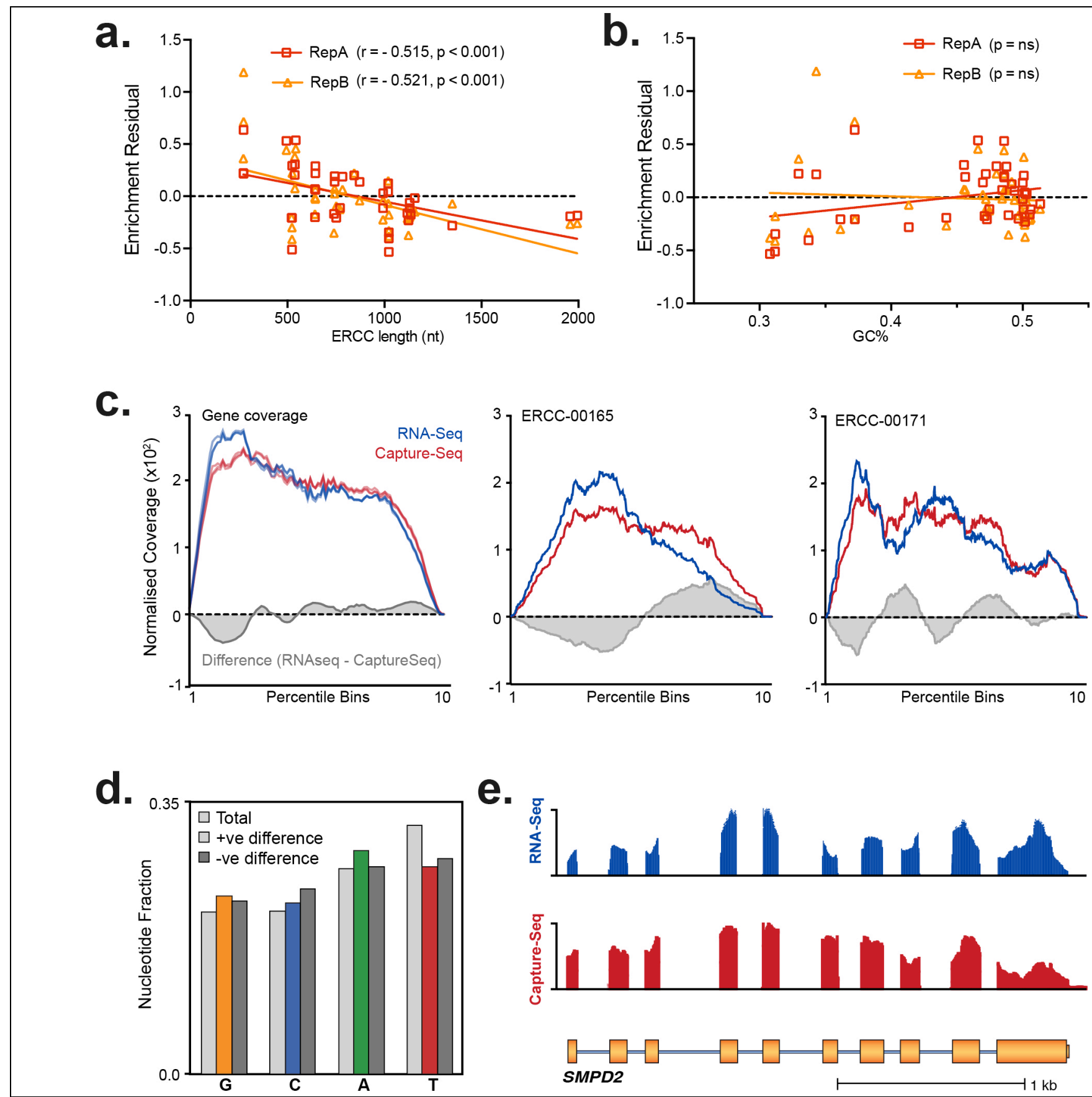

\section{Supplementary Figure 4}

\section{Comparative analysis of technical bias between RNA sequencing and CaptureSeq}

(a,b) Relationship between ERCC length (a), GC\% (b) and CaptureSeq performance on moderately expressed probes compared to RNAseq (enrichment residuals shown). Spearman correlation shown while line is non-linear regression fit. RepA and RepB are technical replicate capture hybridizations containing three biological replicate samples. (c) Combined sequence read coverage across ERCC all standards merged (left) or two representative ERCC controls (middle, right) by RNAseq (blue) and CaptureSeq (red) Difference between read coverage indicated by grey shaded area. (d) Relative nucleotide enrichment for ERCC sequences that exhibit differential coverage between RNAseq and CaptureSeq. No 
specific nucleotide bias is observed in regions exhibiting differential coverage. (e) Sequenced read coverage profile of SMPD2 gene by RNAseq (blue) and CaptureSeq (red). Only minor variation is observed between the two profiles. 

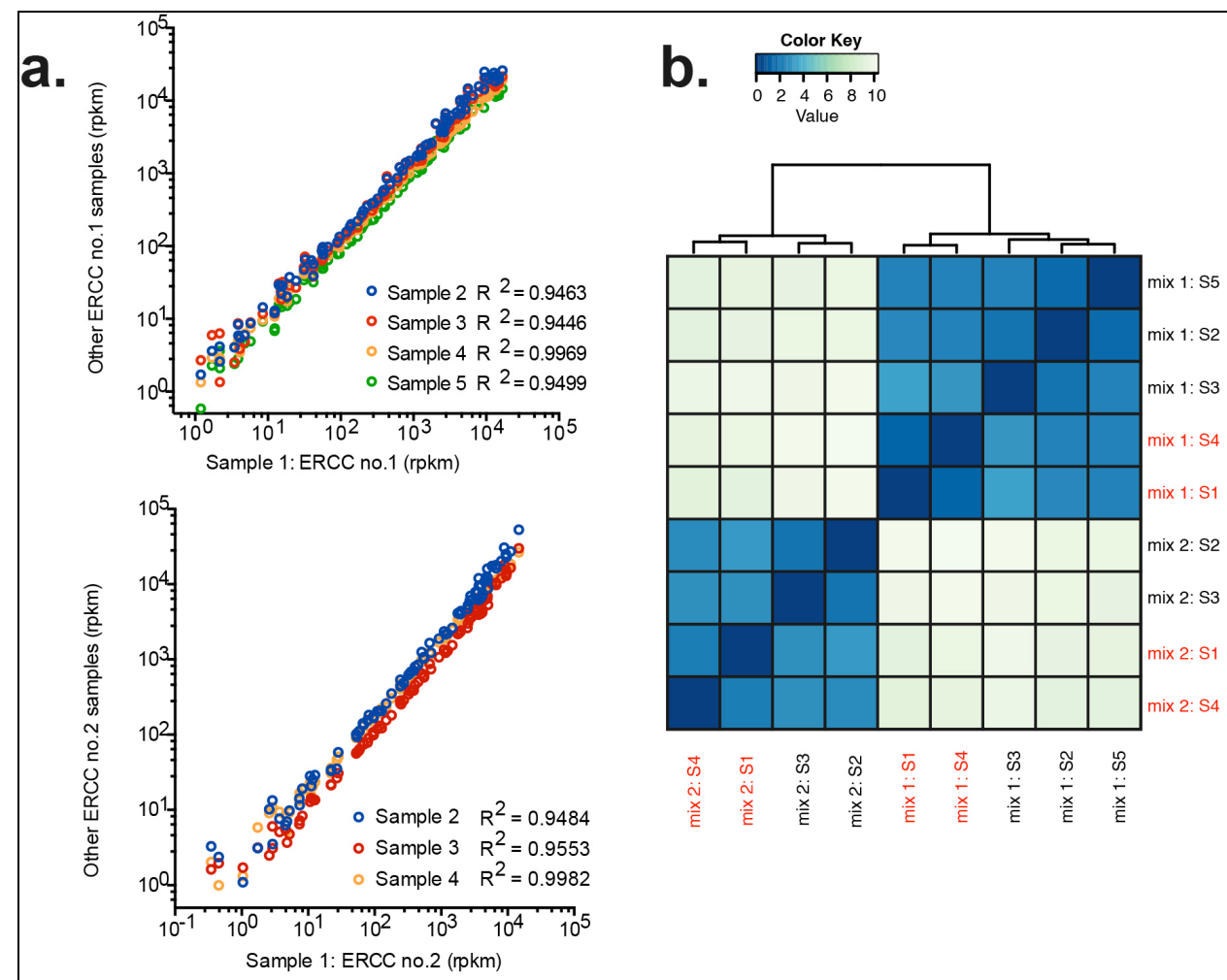

e.

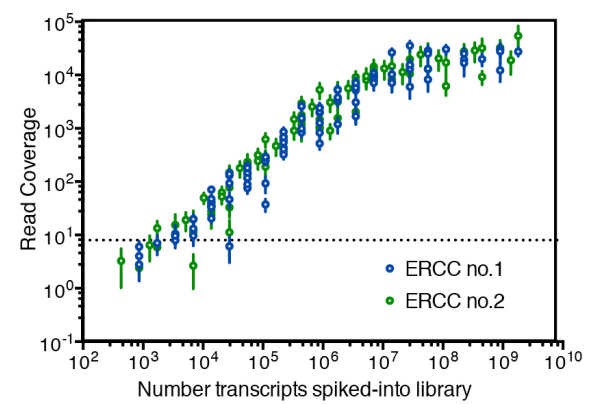

C.
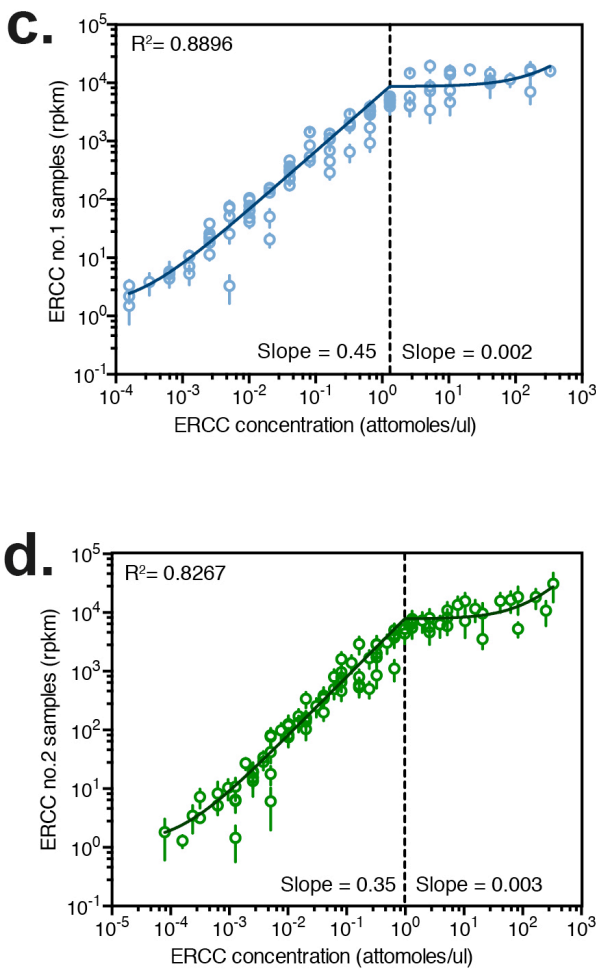

\section{Supplementary Figure 5}

\section{Analysis of differential gene expression between samples with CaptureSeq}

(a) Pearson correlations of measured abundance of ERCC probes for one representative sample vs. all others containing the same ERCC mix, (top) ERCC Mix no.1 (bottom) ERCC Mix no.2. Two multiplexed capture hybridizations were performed containing a mix of ERCC no.1 and no.2 samples. Slightly higher correlations equate to samples present in same hybridization. (b) Clustering of ERCC read counts following variance stabilizing transformation. ERCC no.1 ( $n=5$ ) and no. $2(n=4)$ clearly separate followed by separation by capture hybridization. Samples present in same hybridization shown in red and black respectively. (c,d) Relationship between ERCC concentration and detected ERCC abundance. Segmental linear regression to determine the ERCC concentration at which saturation occurs (dotted line). Error bars are s.d. Linear slopes from segmental linear regression and the Pearson correlation for non-saturating concentrations are provided (c) ERCC no1 samples $n=5$ biological replicates. Saturation at 1.30 attomoles $/ \mu$ (d) ERCC no2 samples $n=4$ biological replicates. Saturation at 0.976 attomoles/ $\mu$ l. (e) Averaged read coverage for each ERCC probe from ERCC no. 1 $(n=5)$ and ERCC no2 ( $n=4)$ pools. Error bars are s.d. Y-axis dotted line shows 8 fold coverage. (f) edgeR MA plot of log fold change for each ERCC control between the two mixes against transcript expression in log CPM (counts per million). 
Differentially expressed (DE) controls coloured red, non-DE coloured black. Zero fold change between two samples shown by blue line. edgeR performed using TMM normalization. 


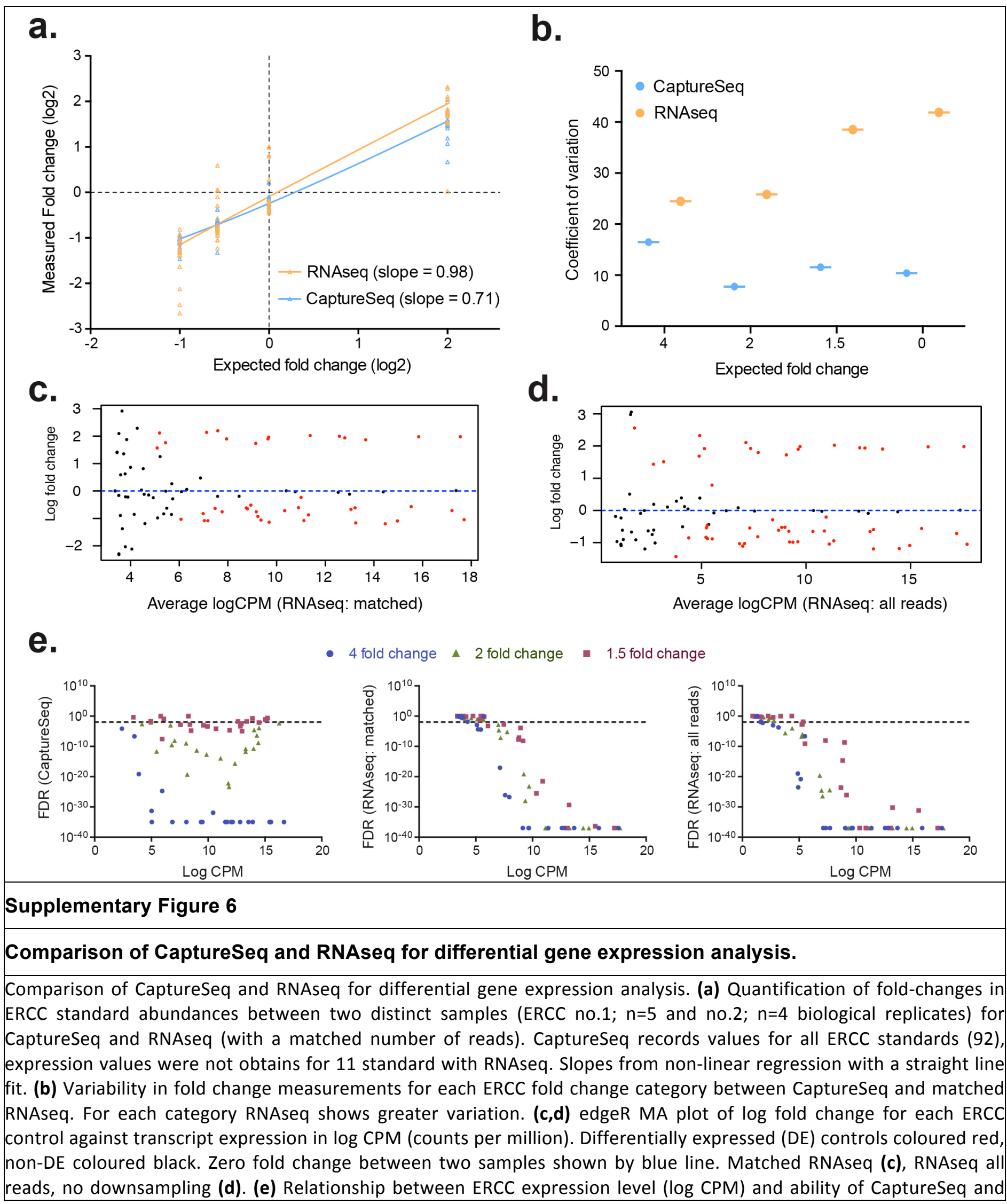


RNAseq to detect $D E$, given various levels of expression differences between two groups. (left) CaptureSeq, (middle) Matched RNAseq, (right) RNAseq all reads, no downsampling. FDR: false discovery rate. 1\% FDR shown by dashed line. FDR values limited to minimum value of $10^{\Lambda^{-37}}$. 


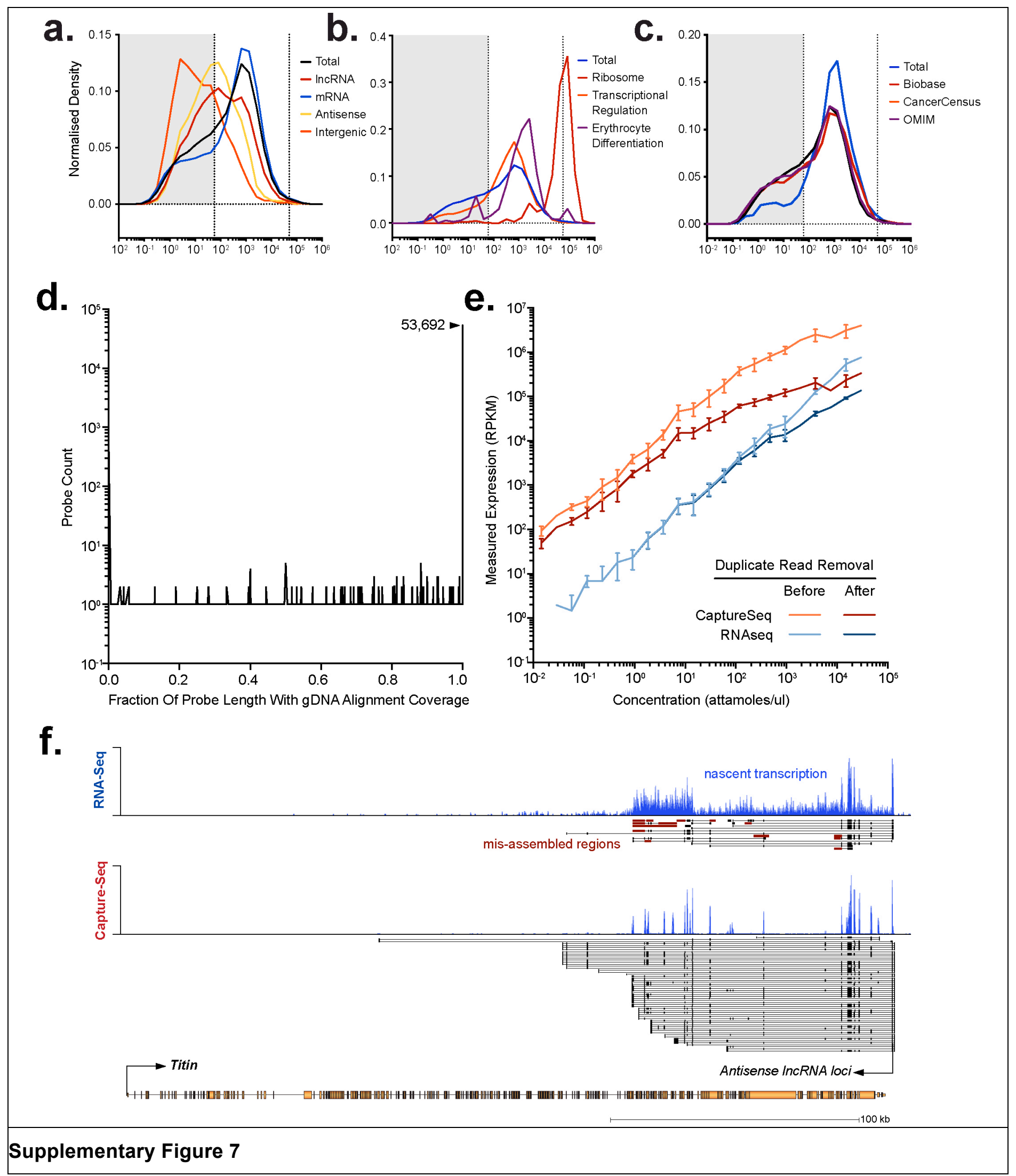




\section{Targeted sequencing of IncRNAs with CaptureSeq}

Frequency distribution of expression for different gene classes according to biotype (a), gene ontology biological function (b) or annotation in disease database (c) in K562 cells. (d) Frequency distribution of probes relative to fraction of length with overlapping alignments from captured genomic DNA. We find $>1$-fold coverage across the entirety of 96.5\% of probes, thereby validating the ability to capture gDNA. (e) Plot showing measured relative to known abundance of ERCC standards by CaptureSeq (orange) and RNAseq (dark blue). We have plotted measured abundance before (orange, light blue) and after (red, dark blue) removing duplicate reads. While removing duplicate reads may reduce the impact of PCR amplification artifacts, it also causes the abundance of ERCC Spike-Ins to be underestimated, decreasing the quantitative range of CaptureSeq and is therefore not recommended. (f) Genome browser view showing read alignment profile and assembled transcripts from RNAseq (upper) and CaptureSeq (lower) across Titin-antisense IncRNA locus. CaptureSeq read alignment shows higher specificity for exons, with less reads derived from nascent transcription present, resulting in more accurate transcript assembly. By contrast, RNAseq shows large amount of nascent transcription, resulting in the mis-assembly of transcripts locus with 'retained' introns. 

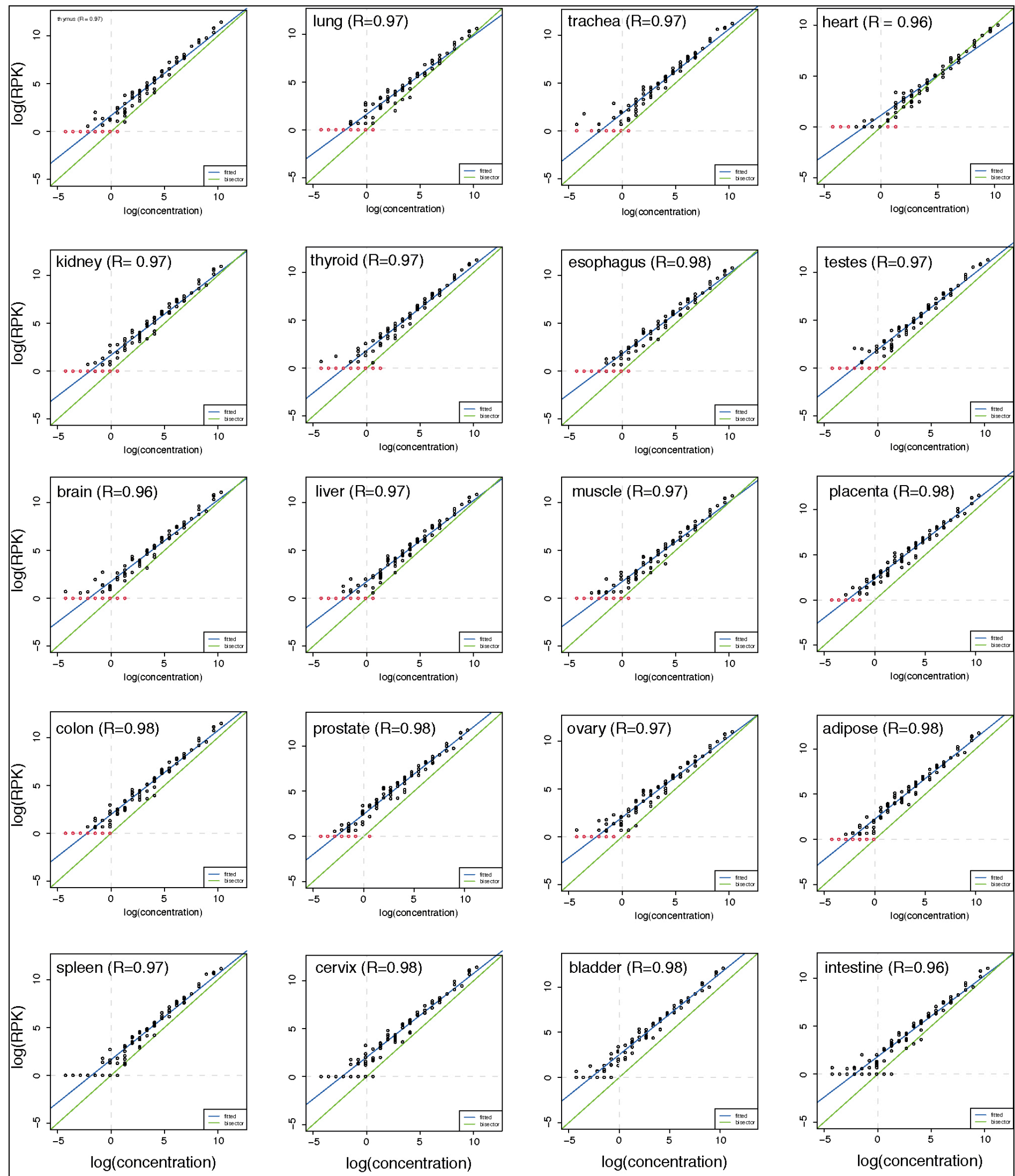


\section{Supplementary Figure 8}

Quantitative accuracy of each tissue within expression atlas

Measured expression (FPKM) of ERCC standards in each human tissue library analyzed. Pearson's correlation indicate quantitative accuracy of libraries following capture. Despite enhanced coverage some ERCC probes (red) remain undetected, indicating sequencing has not proceeded to saturation. 


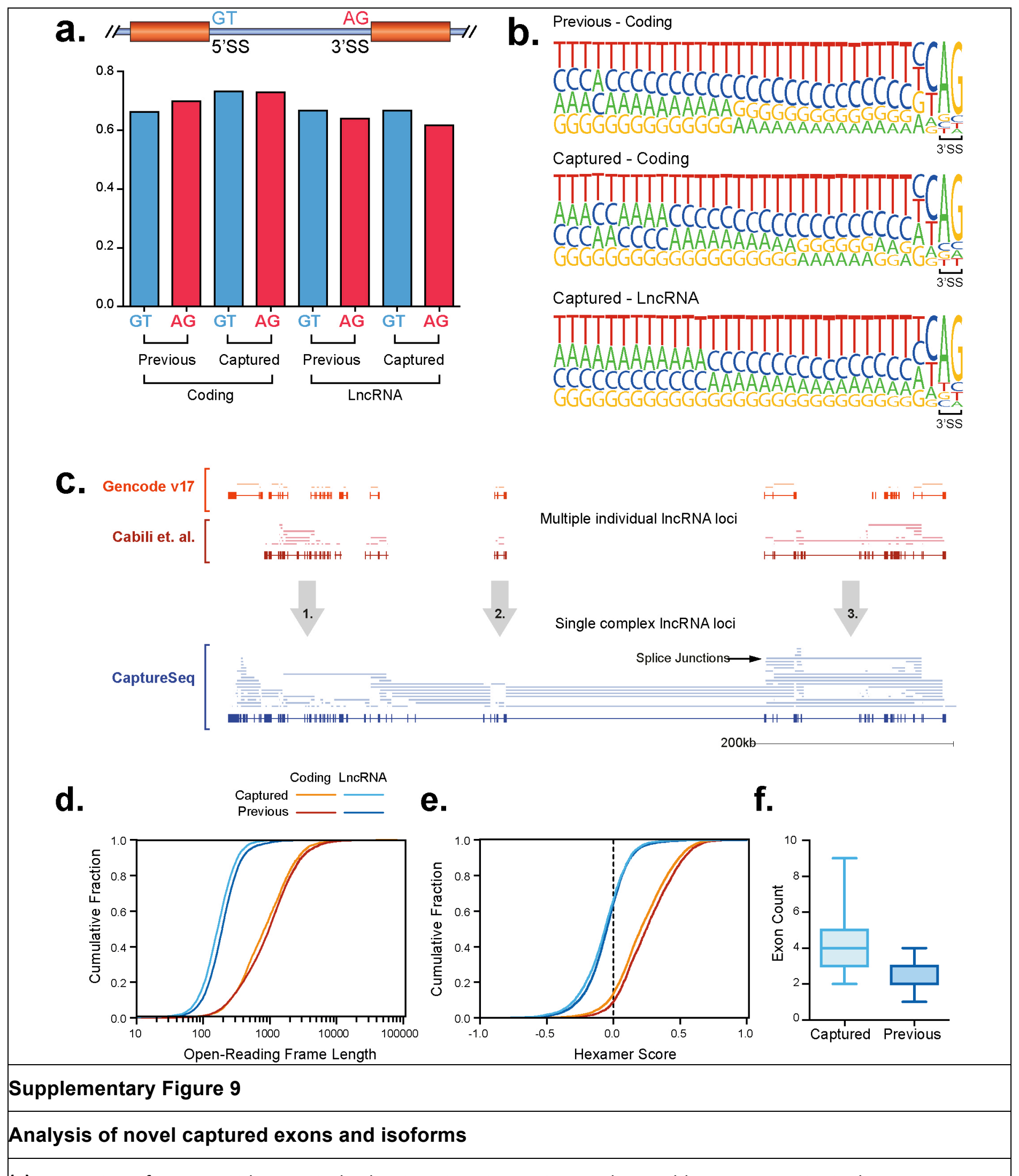

(a) Proportion of introns with canonical splice junctions in previous coding and IncRNA exons is similar to new introns 
identified using CaptureSeq. (b) Sequence motif at 3' intron end shows similar enrichment for poly-pyrimidine tract and splice elements in previously annotated introns and new introns identified by CaptureSeq. (c) Example of multiple previous IncRNA annotations that are merged into single higher-order contiguous IncRNA loci following more complete and accurate assembly with CaptureSeq. $(\mathbf{d}, \mathbf{e})$ Frequency distribution of open-reading frame length and hexamer score indicates distinction between coding and noncoding transcripts analyzed from CaptureSeq assembled transcripts. (f) Box-whisker plot showing CaptureSeq assembled gene models contain more exons and are more complete than previous annotations (based on GENCODE v19, Cabili. et al. (2011), and IncRNAdb) used to design the capture array ${ }^{8-10}$ 


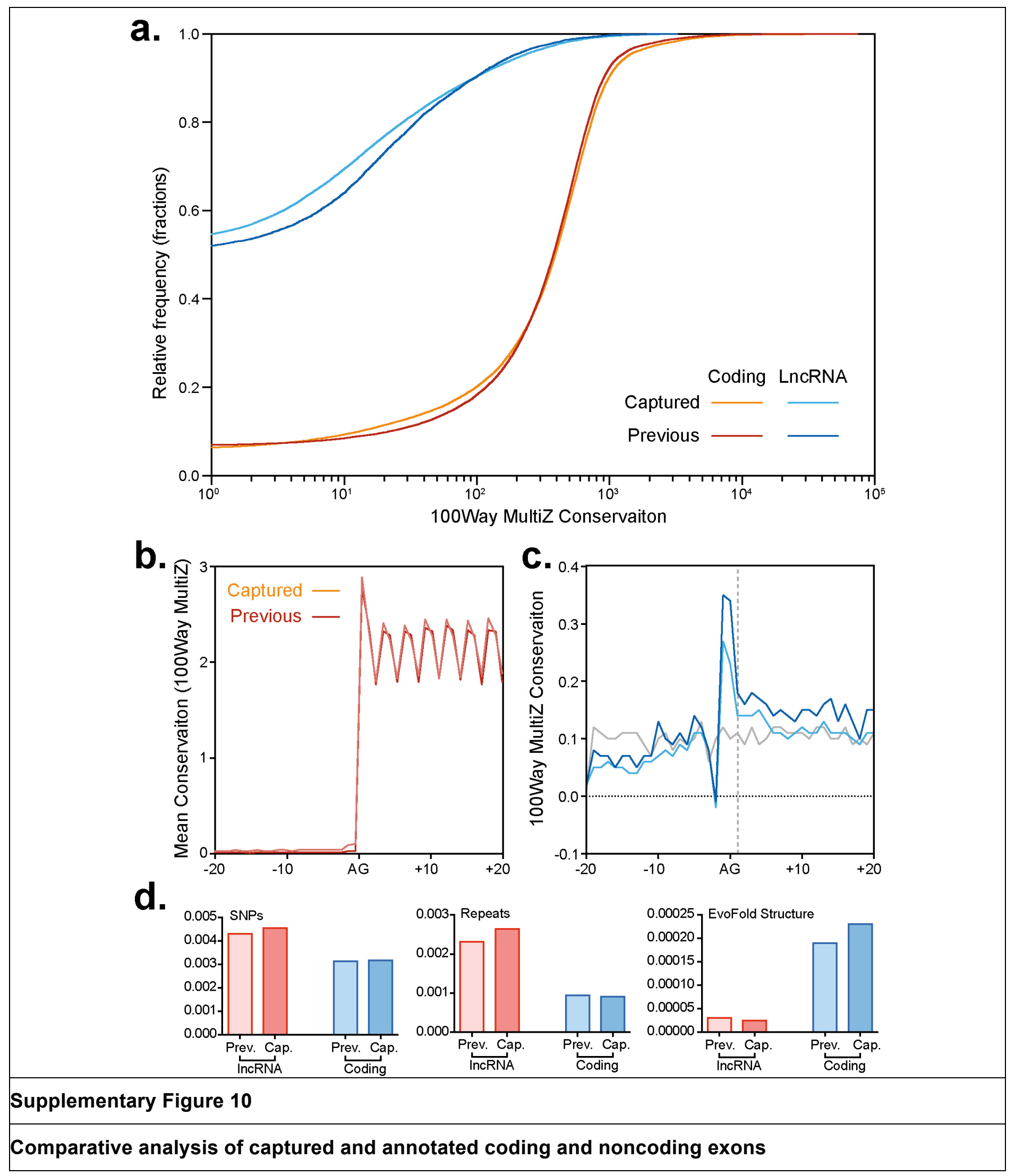


(a) Cumulative frequency distribution indicating the conservation (according to 100-way Mutliz Alignment) of previously annotated (based on GENCODE v19, Cabili. et al., and IncRNAdb) coding and IncRNA exons relative to novel exons identified using CaptureSeq. (b) Conservation at 3' exon boundary showing 3-nt periodicity characteristics of previously known coding gene exons (red) relative to new coding gene exons (orange) identified by CaptureSeq and (c) similar conservation of splice elements in previous IncRNA annotations relative to new IncRNA exons identified by CaptureSeq. (d) Comparison of SNP, repeat and predicted RNA secondary structure density between previous gene annotations (based on GENCODE v19, Cabili. et al. (2011), and IncRNAdb) ${ }^{8-10}$ and new annotations assembled from CaptureSeq experiments. 
a.

н

H

$\underset{111+1}{11+1}$

(1)

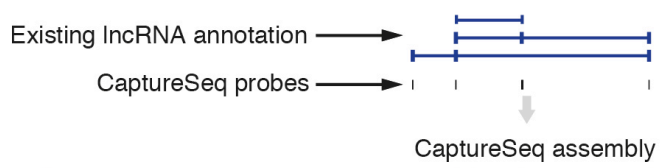

CaptureSeq assembly
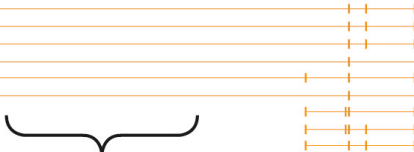

CaptureSeq IncRNA assembly

Chr11 87,100,000

$200 \mathrm{~kb}$

hg19

$87,650,000$

b.

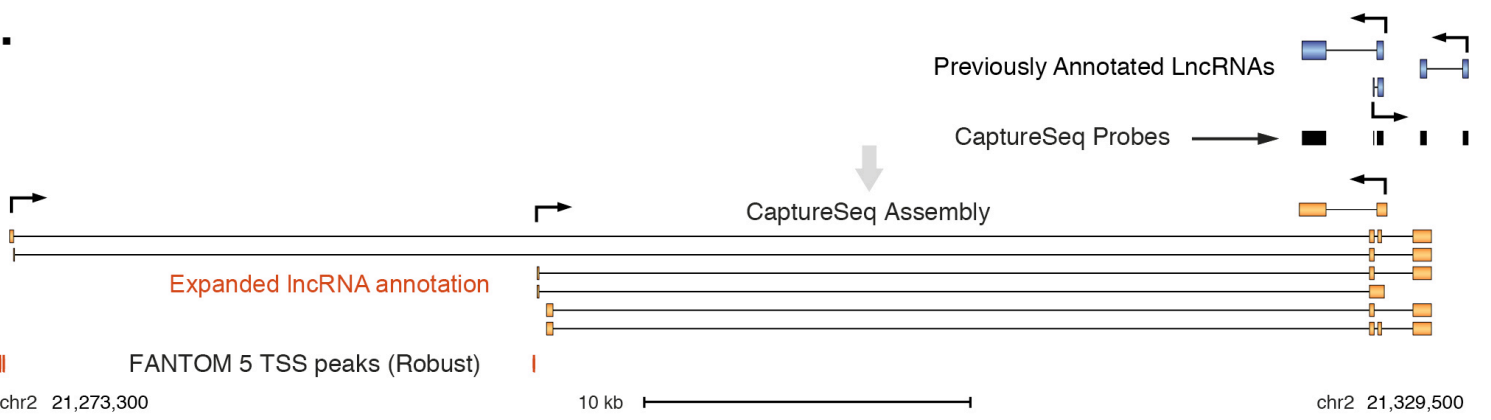

c.
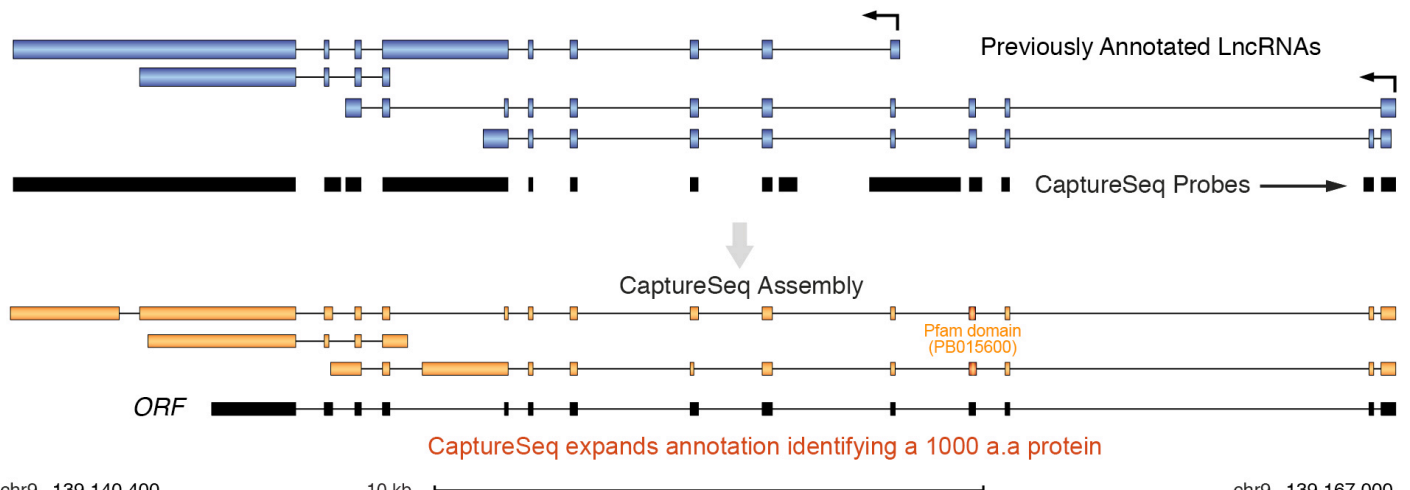

d.
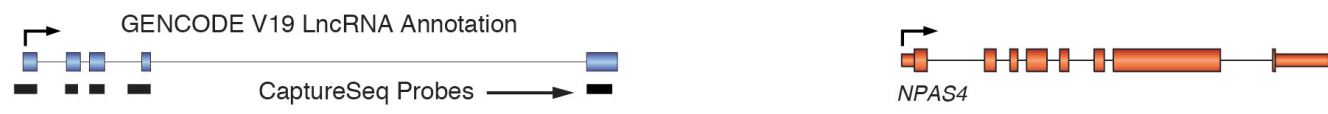

NPAS4

CaptureSeq Assembly
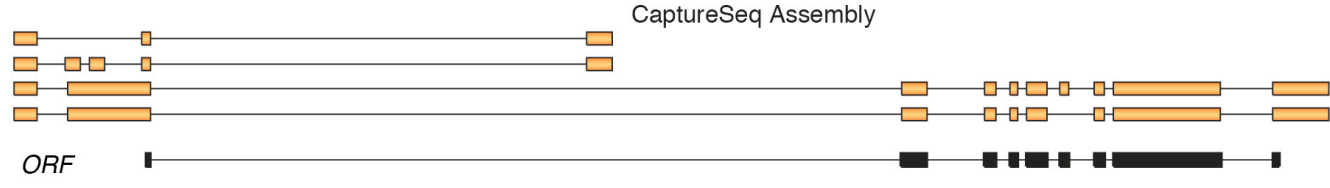

hr11 66,176,500

$10 \mathrm{~kb}$

$66,194,500$ 


\section{Supplementary Figure 11}

\section{Examples of transcripts assembled following CaptureSeq.}

(a) Targeting previous IncRNA annotations (blue) integrates them into a single complex locus. (b) CaptureSeq ensnares additional novel exons into the initial annotation, thereby expanding the previous annotation to annotated TSS's. (c) CaptureSeq revises previous IncRNA annotations to identify a 1021 amino acid ORF. An assembly gap in GRCh37 (hg19) means the protein N-terminal may not be present. A new contig in GRCh38 places MGC50722 6kb upstream suggesting the possibility these two loci form one gene. (d) LncRNA can be erroneously annotated when only transcript fragments are available as demonstrated in example showing a IncRNA locus contains distal coding exons for downstream NPAS4 gene. Arrows indicate direction of transcription. Fantom $5^{11}$ TSS on forward strand (red) and reverse strand (blue). 


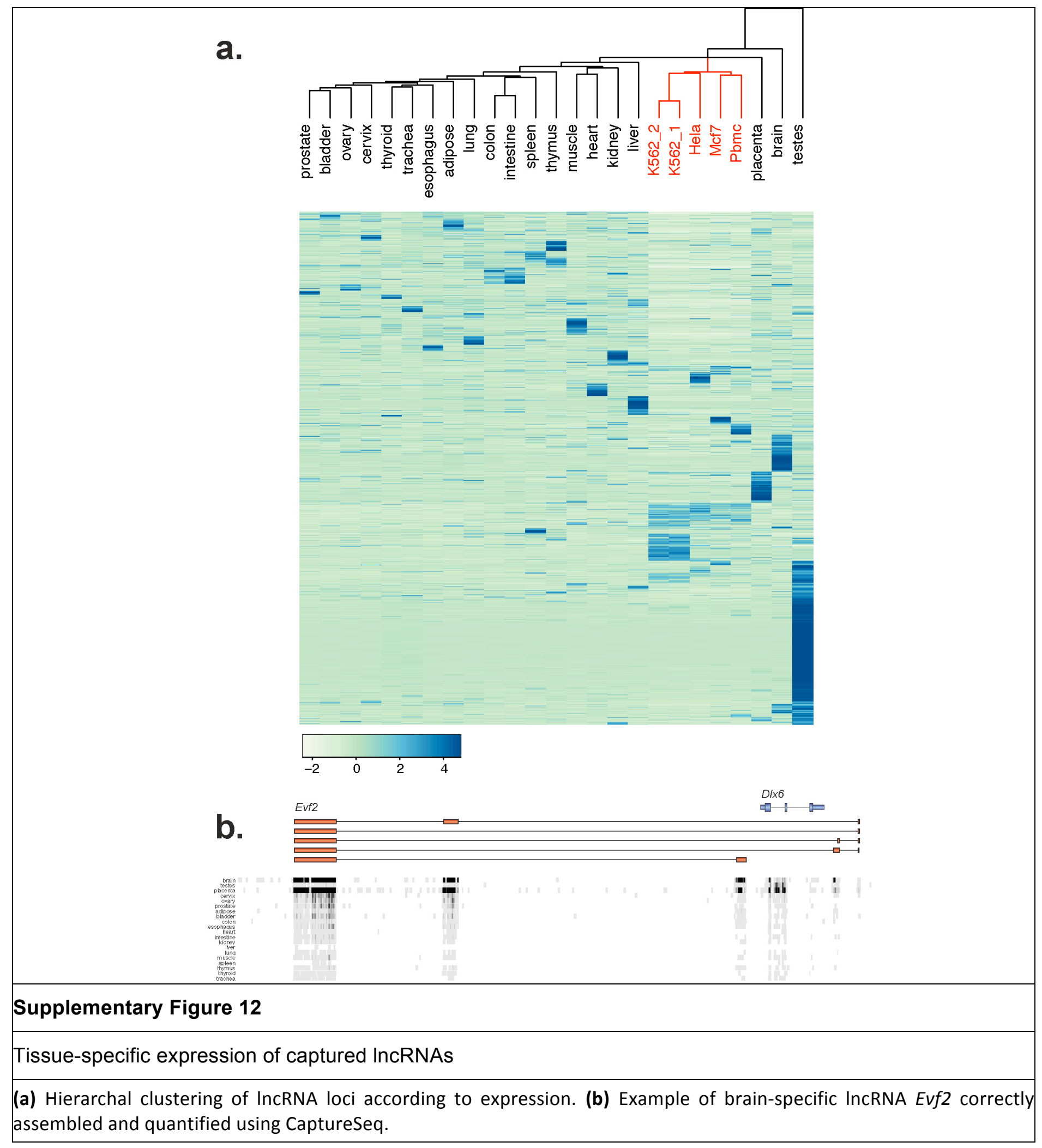


a.

CaptureSeq Probe

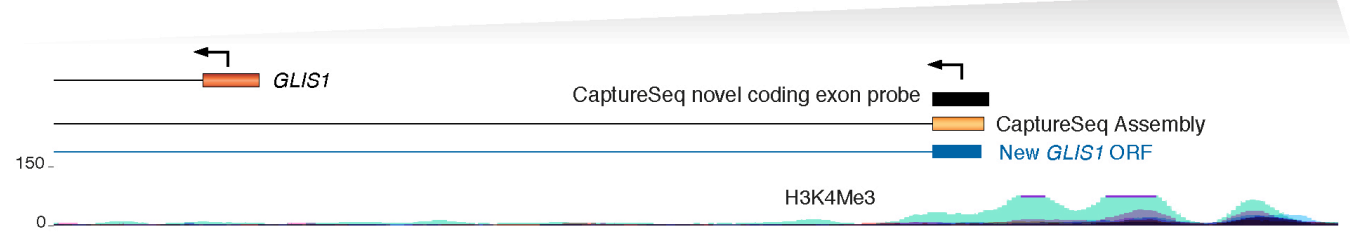

b.
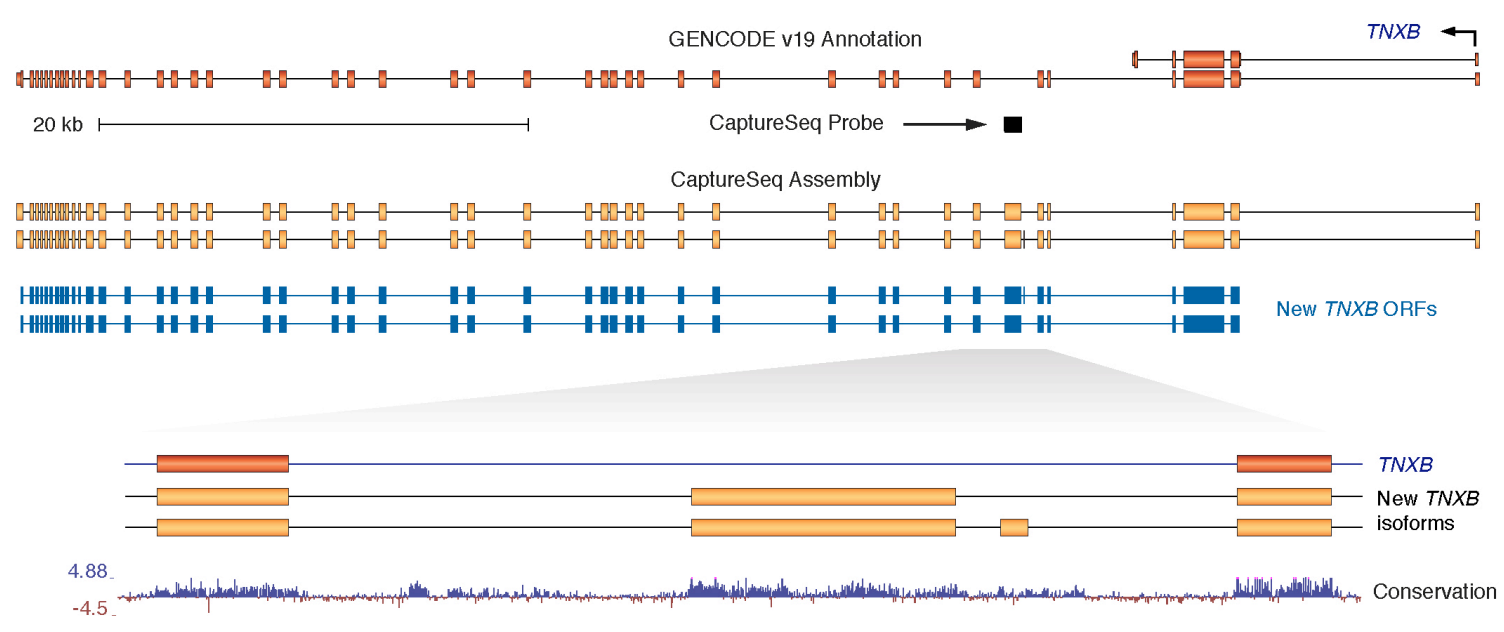

C.

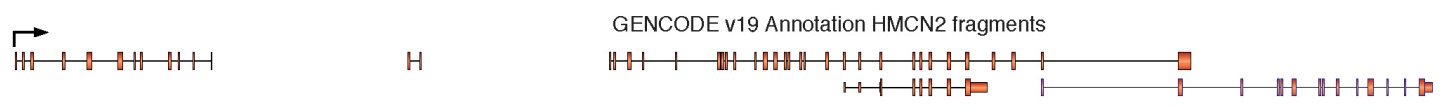

I | I || | I I I || I I I II I Novel Exons (Lindblad-Toh et al. 2011) targetted by CaptureSeq

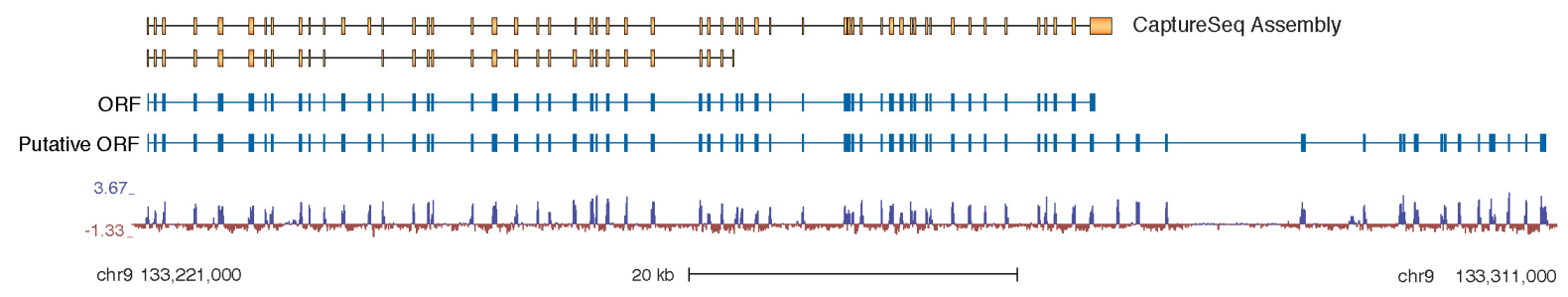

d.

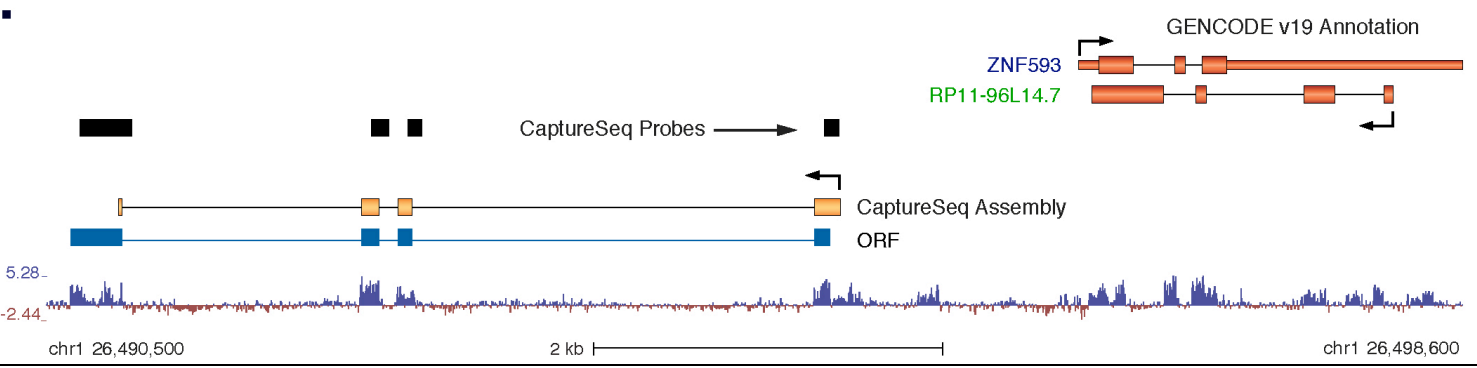




\section{Supplementary Figure 13}

\section{Examples of captured novel coding exons}

Examples of novel coding exons within GENCODE genes assembled following CaptureSeq. (a) Identification of a novel transcription start site for GLIS1 gene well supported by chromatin marks for transcriptional initiation. New first exon adds 175 amino acids to $5^{\prime}$ of protein. (b) Novel internal coding exons in TNXB. Novel exon(s) are conserved and maintain the TNXB reading frame. (c) Targeting novel exons solely identified by evolutionary conservation enables the identification of novel exons that help assemble multiple HMNC2 annotation fragments into a contiguous gene locus. (d) Putative novel coding locus in bi-directional orientation with ZNF593 contains a 186 amino acid ORF. 